\title{
Some Properties of the $k$-bonacci Words on the Infinite Alphabet
}

\author{
Narges Ghareghani \\ Department of Industrial design, College of Fine Arts, \\ University of Tehran, Tehran, Iran \\ ghareghani@ut.ac.ir, ghareghani@ipm.ir
}

Morteza Mohammad-Noori

School of Mathematics, Statistics and Computer Science, College of Science, University of Tehran, Tehran, Iran

mmnoori@ut.ac.ir

\section{Pouyeh Sharifani}

Institute for Research in Fundamental Sciences (IPM), Tehran, Iran

pouyeh.sharifani@gmail.com

Submitted: Feb 29, 2020; Accepted: Aug 10, 2020; Published: Sep 18, 2020

(C) The authors. Released under the CC BY-ND license (International 4.0).

\begin{abstract}
The Fibonacci word $W$ on an infinite alphabet was introduced in [Zhang et al., Electronic J. Combinatorics 2017 24(2), 2-52] as a fixed point of the morphism $2 i \rightarrow(2 i)(2 i+1),(2 i+1) \rightarrow(2 i+2), i \geqslant 0$. Here, for any integer $k>2$, we define the infinite $k$-bonacci word $W^{(k)}$ on the infinite alphabet as $\varphi_{k}^{\omega}(0)$, where the morphism $\varphi_{k}$ on the alphabet $\mathbb{N}$ is defined for any $i \geqslant 0$ and any $0 \leqslant j \leqslant k-1$, by

$$
\varphi_{k}(k i+j)= \begin{cases}(k i)(k i+j+1) & \text { if } j=0, \cdots, k-2, \\ (k i+j+1) & \text { otherwise. }\end{cases}
$$

We consider the sequence of finite words $\left(W_{n}^{(k)}\right)_{n \geqslant 0}$, where $W_{n}^{(k)}$ is the prefix of $W^{(k)}$ whose length is the $(n+k)$-th $k$-bonacci number. We then provide a recursive formula for the number of palindromes that occur in different positions of $W_{n}^{(k)}$. Finally, we obtain the structure of all palindromes occurring in $W^{(k)}$ and based on this, we compute the palindrome complexity of $W^{(k)}$, for any $k>2$.
\end{abstract}

Mathematics Subject Classifications: 68R15, 11B50 


\section{Introduction}

Finite and infinite Fibonacci words are among the most studied ones in combinatorics of words and have important roles in computer science, based on their optimal properties and various applications, see for example $[13,3,14,5]$. The sequence of finite Fibonacci words $\left(F_{n}\right)_{n \geqslant-1}$ is given by $F_{-1}=1, F_{0}=0$ and the recurrence relation $F_{n}=F_{n-1} F_{n-2}$ which holds for $n \geqslant 1$. An equivalent way to give these words for $n \geqslant 0$, is using $F_{n}=\psi^{n}(0)$, where $\psi$ is the binary morphism $0 \rightarrow 01,1 \rightarrow 0$. The infinite Fibonacci word is then given by $F_{\infty}=\lim _{n \rightarrow \infty} F_{n}$ or equivalently by $F_{\infty}=\psi^{\omega}(0)$.

The infinite Fibonacci word belongs to the class of infinite aperiodic binary words having the minimal complexity (i.e. the minimal number of factors of each given length); any such word is called a Sturmian word. Sturmian words are well-studied in the literature; they admit some equivalent definitions and have several interesting properties, see $[4,12$, 10] for instance.

A natural extension of finite Fibonacci words to $k$-letter alphabet, $k>2$, is defining finite $k$-bonacci words $\left(F_{n}^{(k)}\right)_{n \geqslant 0}$ by

$$
F_{n}^{(k)}= \begin{cases}0 & \text { if } n=0 \\ F_{n-1}^{(k)} \ldots F_{0}^{(k)} n & \text { if } 1 \leqslant n<k \\ F_{n-1}^{(k)} \ldots F_{n-k}^{(k)} & \text { if } n \geqslant k .\end{cases}
$$

Alternatively, these words may be given by $F_{n}^{(k)}=\psi_{k}^{n}(0)$, for $n \geqslant 0$, where $\psi_{k}:\{0, \ldots, k-$ $1\}^{*} \rightarrow\{0, \ldots, k-1\}^{*}$ is the morphism

$$
\psi_{k}(i)= \begin{cases}0(i+1) & \text { if } i=0, \cdots, k-2, \\ 0 & \text { if } i=k-1\end{cases}
$$

The infinite $k$-bonacci word is then given by $F_{\infty}^{(k)}=\lim _{n \rightarrow \infty} F_{n}^{(k)}$ or equivalently by $F_{\infty}^{(k)}=$ $\psi_{k}^{\omega}(0)$.

While the infinite Fibonacci word is the simplest example of Sturmian words, the infinite $k$-bonacci word is similarly related to the most natural extension of Sturmian words, namely episturmian words. More precisely, the $k$-bonacci word is the simplest example of non-ultimately periodic episturmian words on the $k$-letter alphabet; to see the definition and properties of episturmian words see $[6,4,11,8,7]$.

The infinite Fibonacci word over the infinite alphabet of nonnegative integers, $\mathbb{N}$, denoted here as $W^{(2)}$, is presented in [15] as the fixed point of the morphism $\varphi_{2}$ starting with 0 , where $\varphi_{2}$ is given by $\varphi_{2}(2 i)=(2 i)(2 i+1)$ and $\varphi_{2}(2 i+1)=2 i+2$ for $i \geqslant 0$. More precisely, we have $W^{(2)}=\varphi_{2}^{\omega}(0)$. The authors of [15] have also studied the finite Fibonacci words over $\mathbb{N}$, namely the words $W_{n}^{(2)}=\varphi_{2}^{n}(0)$. It is obvious that when the digits of $W_{n}^{(2)}$ and $W^{(2)}$ are calculated $\bmod 2$, these words are reduced to $F_{n}$ and $F_{\infty}$, respectively. Among several properties of words $W_{n}^{(2)}$ and $W^{(2)}$ studied in [15], the authors characterized palindromic factors of $W_{n}^{(2)}$ and $W^{(2)}$. Particularly, the authors showed that in contrast to the ordinary infinite Fibonacci word which contains palindromic factors of 
arbitrary length, the word $W^{(2)}$ has no palindrome of length greater than 3 . Some more properties of these words were consequently studied by Glen et al. in [9]. Among other results, they computed the number of palindromes in $W_{n}^{(2)}$.

In this paper, we introduce finite and infinite $k$-bonacci words on the infinite alphabet $\mathbb{N}$, denoted respectively as $W_{n}^{(k)}$ and $W^{(k)}$. Studying theses words, we characterize the palindromic factors of $W^{(k)}$ for any fixed integer $k \geqslant 3$. More precisely we show that the length of a palindromic factor of $W^{(k)}$ belongs to the set $L_{k}=\{2\} \cup\{2 i-1: 2 \leqslant i \leqslant$ $\left.3.2^{k-2}\right\}$. Conversely, for each element $\ell$ of $L_{k}$ we give the structure of palindromes of $W^{(k)}$ with length $\ell$. We also enumerate the total number of palindromes of $W_{n}^{(k)}$.

\section{Definitions and notation}

In this paper, the alphabet, which can be a finite or a countable infinite set, is denoted as $\mathcal{A}$. When the alphabet is infinite, we simply take $\mathcal{A}=\mathbb{N}$. Each element of the alphabet $\mathcal{A}$ is called a letter. When $\mathcal{A}=\mathbb{N}$, we equivalently use the term digit instead of letter. We denote by $\mathcal{A}^{*}$ the set of finite words over $\mathcal{A}$ and we let $\mathcal{A}^{+}=\mathcal{A}^{*} \backslash\{\epsilon\}$, where $\epsilon$ is the empty word. We denote by $\mathcal{A}^{\omega}$ the set of all infinite words over $\mathcal{A}$ and we let $\mathcal{A}^{\infty}=\mathcal{A}^{*} \cup \mathcal{A}^{\omega}$. If $a \in \mathcal{A}$ and $W \in \mathcal{A}^{\infty}$, then the symbols $|W|$ and $|W|_{a}$ denote respectively the length of $W$, and the number of occurrences of letter $a$ in $W$ (It is obvious that when $W \in \mathcal{A}^{\omega}$, $|W|=\infty)$. For any word $W \in \mathcal{A}^{\infty}, \mathcal{A l p h}(W)$ is defined to be the set of letters which have at least one occurrence in $W$, that is $\mathcal{A l p h}(W)=\left\{a \in \mathcal{A}:|W|_{a}>0\right\}$.

A word $V \in \mathcal{A}^{*}$ is a factor of a word $W \in \mathcal{A}^{\infty}$, denoted as $V \prec W$, if there exist $U \in \mathcal{A}^{*}$ and $U^{\prime} \in \mathcal{A}^{\infty}$, such that $W=U V U^{\prime}$. A word $V \in \mathcal{A}^{*}$ (resp. $V \in \mathcal{A}^{\infty}$ ) is said to be a prefix (resp. suffix) of a word $W \in \mathcal{A}^{\infty}$, denoted as $V \triangleleft W$ (resp. $V \triangleright W$ ), if there exists $U \in \mathcal{A}^{\infty}$ (resp. $U \in \mathcal{A}^{*}$ ) such that $W=V U$ (resp. $W=U V$ ). We denote the

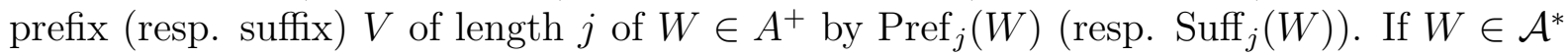
and $W=V U$ (resp. $W=U V$, ) we merely write $V=W U^{-1}\left(\operatorname{resp} . \quad V=U^{-1} W\right)$. For a finite word $W=w_{1} w_{2} \ldots w_{n}$, with $w_{i} \in \mathcal{A}$ and for $1 \leqslant j \leqslant j^{\prime} \leqslant n$, we denote $W\left[j, j^{\prime}\right]=w_{j} \ldots w_{j^{\prime}}$, and for simplicity we denote $W[j, j]$ by $W[j]$. The reversal of a finite word $W=w_{1} w_{2} \ldots w_{n}$, with $w_{i} \in \mathcal{A}$, is $W^{R}=w_{n} w_{n-1} \ldots w_{1}$. A word $W \in \mathcal{A}^{*}$ is called palindrome if $W=W^{R}$. The set of all palindromic factors of the word $W \in \mathcal{A}^{\infty}$ is denoted by $\operatorname{Pal}(W)$. When the alphabet is finite, for any word $U \in \mathcal{A}^{\infty}$, the number of palindromic factors of length $n$ of $U$, called the palindrome complexity of $U$, is denoted by $\operatorname{pal}_{U}(n)$ (for more information about the palindrome complexity see $[1,2]$ and the references therein). When the alphabet is infinite (i.e. $\mathcal{A}=\mathbb{N}$ ), the definition of palindrome complexity can naturally be extended to all words $U \in \mathcal{A}^{\infty}$ with the same notation.

Let $P$ be a palindrome of odd length $2 k+1$ and $W=\operatorname{Pref}_{k}(P)$. Then the letter $a \in \mathcal{A}$ satisfying $P=W a W^{R}$, is called the center of the palindrome $P$. If $P$ is a palindrome of even length, then the center of $P$ is defined to be the empty word. For any occurrence of a palindromic factor $P \in \mathcal{A}^{*}$ in a word $W \in \mathcal{A}^{\infty}$ such as $W=U P V$ with $U \in \mathcal{A}^{*}, V \in \mathcal{A}^{\infty}$, the center position of this occurrence of $P$ in $W$ is denoted by $c_{p}(P, W)$ and defined to be $|U|+\frac{|P|+1}{2}$. We notice that when $|P|$ is even the center-position is a non-integer. A 
palindromic factor $P \in \mathcal{A}^{*}$ of $W \in \mathcal{A}^{\infty}$ is called a maximal palindromic factor of $W$ if there is no longer palindromic factor of $W$ with the same center position. We note that a maximal palindromic factor of a word $W$, could be a factor of another palindromic factor $P$ of $W$. For instance, the only maximal palindromic factors of $W=1213121$ are 1,121 and 1213121.

For $1 \leqslant i \leqslant n$, let $U_{i} \in \mathcal{A}^{*}$; then $\prod_{i=n}^{1} U_{i}$ is defined to be $U_{n} U_{n-1} \ldots U_{1}$. For a finite word $W$ and an integer $n, n \oplus W$ denotes the word obtained by adding $n$ to each letter of $W$. For example, let $W=01023$ and $n=5$, then $n \oplus W=56578$. For a finite set $S=\left\{S_{1}, \ldots, S_{m}\right\} \subset \mathcal{A}^{+}$, we define $n \oplus S$ to be the set $\left\{n \oplus S_{1}, \ldots, n \oplus S_{m}\right\}$.

For any integer $k \geqslant 2$ the sequence of $k$-bonacci numbers, denoted by $\left\{f_{n}^{(k)}\right\}_{n \geqslant 0}$, is given as

$$
f_{n}^{(k)}= \begin{cases}0 & \text { if } n=0, \ldots, k-2, \\ 1 & \text { if } n=k-1, \\ \sum_{i=n-k}^{n-1} f_{i}^{(k)} & \text { if } n \geqslant k\end{cases}
$$

The last recurrence relation which holds eventually, states that the $n$-th term of the sequence is the summation of the $k$ previous ones. This reminds the Fibonacci and Tribonacci recurrence relations in the special cases $k=2$ and $k=3$. In fact, $f_{n}^{(2)}$ and $f_{n}^{(3)}$ are essentially the well-known Finonacci and Tribonacci numbers. It is easy to prove that regardless of the first $k-1$ zero terms, the above sequence of $k$-bonacci numbers can be given as

$$
f_{n}^{(k)}= \begin{cases}1 & \text { if } n=k-1, \\ 2^{n-k} & \text { if } k \leqslant n \leqslant 2 k-1, \\ 2 f_{n-1}^{(k)}-f_{n-k-1}^{(k)} & \text { if } n \geqslant 2 k .\end{cases}
$$

We define the finite (resp. infinite) $k$-bonacci words $W_{n}^{(k)}$ (resp. $\left.W^{(k)}\right)$ on the infinite alphabet $\mathbb{N}$, using the morphism $\varphi_{k}$ given below for integers $i \geqslant 0$ and $0 \leqslant j<k$,

$$
\varphi_{k}(k i+j)=\left\{\begin{array}{cl}
(k i)(k i+j+1) & \text { if } j=0, \ldots, k-2 \\
(k i+j+1) & \text { if } j=k-1
\end{array}\right.
$$

More precisely, $W_{n}^{(k)}=\varphi_{k}^{n}(0)$ and $W^{(k)}=\varphi_{k}^{\omega}(0)$ (Note that $W_{0}^{(k)}=F_{0}^{(k)}=0$ ). Consequently $F_{n}^{(k)}=W_{n}^{(k)} \bmod k$, that is for a fixed value of $k$, the $k$-bonacci words over the infinite alphabet are reduced to $k$-bonacci words over a finite alphabet when the digits are calculated $\bmod k$. It can be shown that for $n \geqslant 0$,

$$
\left|F_{n}^{(k)}\right|=\left|W_{n}^{(k)}\right|=f_{n+k}^{(k)} .
$$

We end this section with two examples giving some initial $k$-bonacci words on finite and infinite alphabets.

Example 1. In this example the 3-bonacci words on finite and infinite alphabet are given when $n \leqslant 5$. The infinite Tribonacci words on finite and infinite alphabet are also shown.

$$
W_{0}^{(3)}=0, \quad F_{0}^{(3)}=0,
$$




$$
\begin{array}{ll}
W_{1}^{(3)}=01, & F_{1}^{(3)}=01 \\
W_{2}^{(3)}=0102, & F_{2}^{(3)}=0102, \\
W_{3}^{(3)}=0102013, & F_{3}^{(3)}=0102010 \\
W_{4}^{(3)}=0102013010234, & F_{4}^{(3)}=0102010010201, \\
W_{5}^{(3)}=010201301023401020133435, & F_{5}^{(3)}=010201001020101020100102
\end{array}
$$

The first terms of $W^{(3)}$ and $F_{\infty}^{(3)}$ are as below

$$
\begin{gathered}
W^{(3)}=01020130102340102013343501020130102343435346 \ldots \\
F_{\infty}^{(3)}=01020100102010102010010201020100102010102010 \ldots
\end{gathered}
$$

Example 2. In this example we fix $n=6$ and consider different cases $k=3,4,5,6$.

$$
\begin{aligned}
& W_{6}^{(3)}=01020130102340102013343501020130102343435346, \\
& F_{6}^{(3)}=01020100102010102010010201020100102010102010 . \\
& W_{6}^{(4)}=01020103010201401020103010245010201030102014010201034546, \\
& F_{6}^{(4)}=01020103010201001020103010201010201030102010010201030102 . \\
& W_{6}^{(5)}=0102010301020104010201030102015010201030102010401020103010256, \\
& F_{6}^{(5)}=0102010301020104010201030102010010201030102010401020103010201 . \\
& W_{6}^{(6)}=010201030102010401020103010201050102010301020104010201030102016, \\
& F_{6}^{(6)}=010201030102010401020103010201050102010301020104010201030102010 .
\end{aligned}
$$

\section{Some properties of $W_{n}^{(k)}$}

In this section, we give some recursive identities which state the word $W_{n}^{(k)}$ as the concatenation of previous words of the same type. These identities will help us to discover the structure of palindromes in $k$-bonacci words in the future sections. First we present a simple lemma stating a property of the morphism $\varphi_{k}$ which can be easily deducted from the definition.

Lemma 3. For any integer $i \geqslant 0, \varphi_{k}(k+i)=k \oplus \varphi_{k}(i)$.

Lemma 4. For $1 \leqslant n \leqslant k-1$,

$$
W_{n}^{(k)}=\prod_{i=n-1}^{0} W_{i}^{(k)} n .
$$


Proof. We use induction on $n$. It is easy to check that the statement is true for $n=1$. Suppose the lemma is true for all $i$ with $1 \leqslant i \leqslant n<k-1$. Then

$$
\begin{aligned}
W_{n+1}^{(k)} & =\varphi_{k}\left(W_{n}^{(k)}\right) \\
& =\varphi_{k}\left(\prod_{i=n-1}^{0} W_{i}^{(k)} n\right) \\
& =\prod_{i=n-1}^{0} \varphi_{k}\left(W_{i}^{(k)}\right) \varphi_{k}(n) \\
& =\prod_{i=n}^{1} W_{i}^{(k)} 0(n+1) \quad \text { since } n<k-1 \text { and } \varphi_{k}(n)=0(n+1) \\
& =\prod_{i=n}^{0} W_{i}^{(k)}(n+1) .
\end{aligned}
$$

Lemma 5. For $1 \leqslant n \leqslant k-1$,

$$
W_{n}^{(k)}=W_{n-1}^{(k)} W_{n-1}^{(k)}(n-1)^{-1} n .
$$

Proof. By Lemma 4, we have

$$
\begin{aligned}
W_{n}^{(k)} & =W_{n-1}^{(k)} W_{n-2}^{(k)} \cdots W_{0}^{(k)} n \\
& =W_{n-1}^{(k)} W_{n-1}^{(k)}(n-1)^{-1} n
\end{aligned}
$$

In the next lemma we give a recursive formula for $W_{n}^{(k)}$ when $n \geqslant k$.

Lemma 6. For $n \geqslant k$,

$$
W_{n}^{(k)}=\prod_{i=n-1}^{n-k+1} W_{i}^{(k)}\left(k \oplus W_{n-k}^{(k)}\right) .
$$

Proof. We use induction on $n$. If $n=k$, the statement is true since

$$
\begin{aligned}
W_{k}^{(k)} & =\varphi_{k}\left(W_{k-1}^{(k)}\right) \\
& =\varphi_{k}\left(\prod_{i=k-2}^{0} W_{i}^{(k)}(k-1)\right) \\
& =\prod_{i=k-2}^{0} \varphi_{k}\left(W_{i}^{(k)}\right) \varphi_{k}(k-1) \\
& =\prod_{i=k-1}^{1} W_{i}^{(k)} k \\
& =\prod_{i=k-1}^{1} W_{i}^{(k)}\left(k \oplus W_{0}^{(k)}\right) .
\end{aligned}
$$


Now, suppose Equation (6) holds for all $n$ with $k \leqslant n \leqslant j$; we prove it below for $n=j+1$.

$$
\begin{aligned}
W_{j+1}^{(k)} & =\varphi_{k}\left(W_{j}^{(k)}\right) \\
& =\varphi_{k}\left(\prod_{i=j-1}^{j-k+1} W_{i}^{(k)}\left(k \oplus W_{j-k}^{(k)}\right)\right) \\
& =\prod_{i=j-1}^{j-k+1} \varphi_{k}\left(W_{i}^{(k)}\right) \varphi_{k}\left(k \oplus W_{j-k}^{(k)}\right) \\
& =\prod_{i=j}^{j-k+2} W_{i}^{(k)}\left(k \oplus \varphi_{k}\left(W_{j-k}^{(k)}\right)\right) \text { by Lemma } 3 \\
& =\prod_{i=j}^{j-k+2} W_{i}^{(k)}\left(k \oplus W_{j-k+1}^{(k)}\right) .
\end{aligned}
$$

Corollary 7. For integers $0 \leqslant i \leqslant n, W_{i}^{(k)}$ is a prefix of $W_{n}^{(k)}$.

Proof. By Lemmas 4 and 6, for any integer $n \geqslant 0, W_{n}^{(k)} \triangleleft W_{n+1}^{(k)}$. Hence, the result follows by induction on $n$.

Corollary 8. Let $k>2$. For each $0 \leqslant n \leqslant k-1,\left|W_{n}^{(k)}\right|=2^{n}$ and for each nonnegative $n,\left|W_{n+1}^{(k)}\right| \leqslant 2\left|W_{n}^{(k)}\right|$. Furthermore, the following identity holds.

$$
\left|W_{n+1}^{(k)}\right|=\left\{\begin{array}{cl}
2\left|W_{n}^{(k)}\right| & \text { if } 0 \leqslant n \leqslant k-2, \\
2\left|W_{n}^{(k)}\right|-1 & \text { if } n=k-1, \\
2\left|W_{n}^{(k)}\right|-\left|W_{n-k}^{(k)}\right| & \text { if } n>k-1 .
\end{array}\right.
$$

Proof. Considering the definition of $k$-bonacci numbers in Equation (2) and using Lemmas 4 and 6 , we conclude that $\left|W_{n}^{(k)}\right|=f_{n+k}^{(k)}$ holds for all $n \geqslant 0$. The other statements follow from Equation (3).

Lemma 9. For any $n \geqslant 0$, the digit $n$ is the largest one of $W_{n}^{(k)}$ and appears once at the end of this word.

Proof. We prove this using induction on $n$. When $n=1, W_{1}^{(k)}=\varphi_{k}(0)=01$ and it is obvious that the claim is true. Now suppose the result holds for $n \leqslant m$. We prove it for $n=m+1$ below.

$$
\begin{aligned}
W_{m+1}^{(k)} & =\varphi_{k}\left(W_{m}^{(k)}\right) \\
& =\varphi_{k}\left(W_{m}^{(k)} m^{-1} m\right) \text { by the induction hypothesis } \\
& =\varphi_{k}\left(W_{m}^{(k)} m^{-1}\right) \varphi_{k}(m) .
\end{aligned}
$$

Now, by induction hypothesis all digits of $W_{m}^{(k)} m^{-1}$ are less than $m$. Hence, by definition of $\varphi_{k}$, all digits of $\varphi_{k}\left(W_{m}^{(k)} m^{-1}\right)$ are less than $m+1$. Again using definition of $\varphi_{k}$, the largest digit of $\varphi_{k}(m)$ is $m+1$ which occurs once at the end of $\varphi_{k}(m)$ and the proof is complete. 
The following notation simplifies some definitions and proofs appearing in the rest of the paper. Notation. For any integer $i$, we let $(i)_{*}=\max \{i, 0\}$.

Definition 10. Let $n$ be a positive integer. Considering factorizations of $W_{n}^{(k)}$ given in the Equations (5) and (6), we divide the set of factors of $W_{n}^{(k)}$ into three following types.

- Included factors. The digit $n$ or the factors of $W_{n}^{(k)}$ which are included in any of the words $W_{n-1}^{(k)}, W_{n-2}^{(k)}, \ldots, W_{(n-k+1)_{*}}^{(k)}$ or in $\left(k \oplus W_{n-k}^{(k)}\right)$, if $n \geqslant k$.

- Bordering factors. Factors $F$ which are of the form $F=X_{j} Y_{j}$ for some $(n-k+$ $1)_{*}+1 \leqslant j \leqslant n-1$, where $X_{j} \neq \epsilon$ is a suffix of $W_{j}^{(k)}$ and $Y_{j} \neq \epsilon$ is a prefix of $\prod_{i=j-1}^{(n-k+1)_{*}} W_{i}^{(k)}$. We call any such factor, a bordering factor of type $j$ of $W_{n}^{(k)}$.

- Straddling factors. Factors $F$ which are of the form $F=A B$, where $A \neq \epsilon$ is a suffix of $\prod_{i=n-1}^{(n-k+1)_{*}} W_{i}^{(k)}$ and $B=n$ if $n \leqslant k-1$, and $B$ is a prefix of $k \oplus W_{n-k}^{(k)}$ if $n \geqslant k$; these are called $(A, B)$-straddling factors (or straddling factors for short, if there is no danger of confusion) of $W_{n}^{(k)}$.

Definition 11. Let $n$ be a positive integer. Considering Definition 10, we call a palindromic factor $P$ of $W_{n}^{(k)}$ an included (resp. a bordering, a straddling) palindrome if $P$ is an included (resp. a bordering, a straddling) factor of $W_{n}^{(k)}$.

The following definition helps us to detect factors of length two of $W_{n}^{(k)}$ and will be used in some lemmas.

Definition 12. Let $\mathcal{B}_{1}^{(k)}=\{(a . k) \oplus k i: i \geqslant 0, a \neq 0\}, \mathcal{B}_{2}^{(k)}=\{(0 . b) \oplus k i: i \geqslant 0,1 \leqslant b \leqslant$ $k-1\}$ and $\mathcal{B}_{3}^{(k)}=\{a .0: a \neq 0\}$ and $\mathcal{B}^{(k)}=\mathcal{B}_{1}^{(k)} \cup \mathcal{B}_{2}^{(k)} \cup \mathcal{B}_{3}^{(k)}$.

Lemma 13. Let $B=$ s.t be a factor of length 2 of $W_{n}^{(k)}$, then $B \in \mathcal{B}^{(k)}$.

Proof. By Definition 12, it is easy to see that $\mathcal{B}^{(k)} \oplus k \subset \mathcal{B}^{(k)}$. We prove the lemma by induction on $n$. If $n=1$, then $W_{1}^{(k)}=01$ has just one factor of length 2 , namely $01 \in \mathcal{B}_{2}^{(k)}$. Now let $m>1$ and suppose that the lemma is true for all $n, 1 \leqslant n<m$. To conclude its validity for $m$, let $B$ be a factor of length 2 of $W_{n}^{(k)}$. Considering Definition 10, one of the following cases hold.

- $\boldsymbol{B}$ is an included factor of $\boldsymbol{W}_{\boldsymbol{m}}^{(\boldsymbol{k})}$. Then either $B \prec W_{n}^{(k)}$ for some $(m-k+1)_{*} \leqslant$ $n \leqslant m-1$ or $B \prec\left(k \oplus W_{m-k}^{(k)}\right)$ (this happens only if $\left.m \geqslant k\right)$. The former case leads to $B \in \mathcal{B}^{(k)}$ by the induction hypothesis, while the latter case leads to $B \ominus k \in \mathcal{B}^{(k)}$, whence by $\mathcal{B}^{(k)} \oplus k \subset \mathcal{B}^{(k)}$, we have $B \in \mathcal{B}^{(k)}$.

- $\boldsymbol{B}$ is a bordering factor of $\boldsymbol{W}_{\boldsymbol{m}}^{(\boldsymbol{k})}$. Then by Definition $10, B=j 0$, for some $(n-k+1)_{*}+1 \leqslant j \leqslant n-1$. This means that $B \in \mathcal{B}_{3}^{(k)}$.

- $\boldsymbol{B}$ is a straddling factor of $\boldsymbol{W}_{\boldsymbol{m}}^{(\boldsymbol{k})}$. Then either $B=0 . m$, when $m \leqslant k-1$, or $B=(m-k+1) k$. The latter case leads to $B \in \mathcal{B}_{1}^{(k)}$ by Definition 12 while the former case leads to $B \in \mathcal{B}_{2}^{(k)}$. 
The next corollaries are direct consequences of Lemma 13.

Corollary 14. Let $n \geqslant 0$ and $k>2$. The finite word $W_{n}^{(k)}$ contains no factor 00 .

Corollary 15. Let $s, t \in \mathbb{N}$ and let $n, k$ be two positive integers with $k>2$. If st $\prec W_{n}^{(k)}$, then either $k \mid t$ or $s<t$.

Proof. Suppose that $k V t$, then by Lemma 13, st $\in \mathcal{B}^{(2)}$. Hence, by Definition $12, s<t$.

The following theorem gives us the suffix of length two of the word $W_{n}^{(k)}$.

Theorem 16. Let $n \geqslant 1$ and $j=(n \bmod k)$. Then

- $(n-j) n \triangleright W_{n}^{(k)}$ provided $j \neq 0$.

- $(n-k+1) n \triangleright W_{n}^{(k)}$ provided $j=0$.

Proof. Recall that $W_{n}^{(k)}=\varphi_{k}^{n}(0)$. We prove the theorem using induction on $n$. If $n=j=$ 1 , we have $01 \triangleright \varphi_{k}^{1}(0)$. Suppose that the result holds for all $n, n \leqslant m$. The validity of the result for $n=m+1$ is then proved in the following two cases.

- If $m+1 \equiv 0(\bmod k)$, then $m \equiv k-1(\bmod k)$ and by induction hypothesis $(m-k+1)(m) \triangleright \varphi_{k}^{m}(0)$. Hence,

$$
\begin{array}{r}
\varphi_{k}((m-k+1)(m)) \triangleright \varphi_{k}^{m+1}(0), \\
\varphi_{k}(m-k+1) \varphi_{k}(m) \triangleright \varphi_{k}^{m+1}(0), \\
(m-k+1)(m-k+2)(m+1) \triangleright \varphi_{k}^{m+1}(0) .
\end{array}
$$

- If $m+1 \equiv j(\bmod k)$, and $0<j<k$ then by Lemma $9, m$ is the last digit of $\varphi_{k}^{m}(0)$ or $m \triangleright \varphi_{k}^{m}(0)$. Hence,

$$
\begin{array}{r}
\varphi_{k}(m) \triangleright \varphi_{k}^{m+1}(0) \\
(m-j+1)(m+1) \triangleright \varphi_{k}^{m+1}(0) .
\end{array}
$$

So, the proof is complete.

\section{The number of palindromes in $W_{n}^{(k)}$}

In this section, we are going to count the total number of palindromes in $W_{n}^{(k)}$. Actually, the process of counting all palindromes in $W_{n}^{(k)}$ leads us to find all possible palindromic factors of $W_{n}^{(k)}$. Equations (5) and (6) are essential in the rest of this work. By Equation (5) and Lemma 5, we obtain an explicit formula for the number of palindromes in $W_{n}^{(k)}$ when $n \leqslant k-1$; this is done in Section 4.1. 
Let $P^{(k)}(n)$ denote the total number of palindromes in $W_{n}^{(k)}$ occurring in different positions and $B^{(k)}(n, j)$ and $S^{(k)}(n)$ denote the number of bordering palindromes of type $j$ and straddling palindromes of $W_{n}^{(k)}$, respectively. Then by Definition 11, the following recurrence relation holds

$$
P^{(k)}(n)=\sum_{i=n-k}^{n-1} P^{(k)}(i)+\sum_{i=n-k+2}^{n-1} B^{(k)}(n, i)+S^{(k)}(n) .
$$

The following theorem gives a recurrence formula for computing $P^{(k)}(n)$. The proof which is formally stated in Section 4.4, is based on considering several cases with respect to values of $n$ and $k$. This is done in Sections 4.1-4.3.

Theorem 17. Let $k>2$ and $n \geqslant 1$ be given integers. Then the following holds

(i) If $0 \leqslant n \leqslant k-1$, then $P^{(k)}(n)=n 2^{n-1}+1$,

(ii) If $n \geqslant k$, then $P^{(k)}(n)=\sum_{i=n-k}^{n-1} P^{(k)}(i)+\alpha^{(k)}(n)$, where

$$
\alpha^{(k)}(n)= \begin{cases}2^{k}-k 2^{n-k+2}+n 2^{n-k+1} & \text { if } k \leqslant n \leqslant 2 k-3 \\ 0 & \text { if } n=2 k-2, \\ 2^{n-2 k+2}-1 & \text { if } 2 k-1 \leqslant n \leqslant 3 k-3 \\ 2^{k}-2 & \text { if } n=3 k-2, \\ 0 & \text { if } n>3 k-2 .\end{cases}
$$

To prove the theorem we divide the rest of this section into some subsections with respect to the values of $n$ and $k$.

\subsection{Palindromes in $W_{n}^{(k)}$ when $n \leqslant k-1$}

Lemma 18. For $1 \leqslant n \leqslant k-1, W_{n}^{(k)} n^{-1}$ is a palindromic word.

Proof. We prove this by induction on $n$. Since for every $k>2$ we have $W_{1}^{(k)}=01$, the first step of the induction is true. Suppose $n=j<k-1$, the word $W_{j}^{(k)} j^{-1}$ is a palindrome. Now using Lemma 5, we have

$$
\begin{aligned}
W_{j+1}^{(k)}(j+1)^{-1} & =W_{j}^{(k)} W_{j}^{(k)} j^{-1} \\
& =W_{j}^{(k)} j^{-1} j W_{j}^{(k)} j^{-1},
\end{aligned}
$$

which is a palindromic word by induction hypothesis.

Lemma 19. For every $1 \leqslant n \leqslant k-1, P^{(k)}(n)=2 P^{(k)}(n-1)+2^{n-1}-1$ and $P^{(k)}(0)=1$.

Proof. Since the digit $n$ just occurs in the last position of $W_{n}^{(k)}$, every palindromic factor of $W_{n}^{(k)}$ either equals to $n$ or is a palindromic factor of $W_{n}^{(k)} n^{-1}$. By Lemma 5 , for every $n \leqslant k-1$, we have

$$
W_{n}^{(k)} n^{-1}=W_{n-1}^{(k)} W_{n-1}^{(k)}(n-1)^{-1}=W_{n-1}^{(k)}(n-1)^{-1}(n-1) W_{n-1}^{(k)}(n-1)^{-1} .
$$


From Equation (8), we conclude that $n-1$ occurs once in $W_{n}^{(k)} n^{-1}$. Using Lemma 18 and again using Equation (8), we find that a factor $P$ of $W_{n}^{(k)} n^{-1}$ is a palindromic word if and only if it is either a palindromic factor of $W_{n-1}^{(k)}(n-1)^{-1}$ or $P=a(n-1) a$, where $a$ is a prefix of $W_{n-1}^{(k)}$. Therefore, $P^{(k)}(n)=2\left(P^{(k)}(n-1)-1\right)+\left|W_{n-1}^{(k)}\right|+1$. Hence, using Corollary 8 , we provide $P^{(k)}(n)=2 P^{(k)}(n-1)+2^{n-1}-1$.

Theorem 20. For every $0 \leqslant n \leqslant k-1, P^{(k)}(n)=n 2^{n-1}+1$.

Proof. The proof is easy by induction on $n$.

\subsection{Bordering Palindromes of $W_{n}^{(k)}$}

In this section, we consider the bordering palindromes of $W_{n}^{(k)}$ when $n \geqslant k$. A bordering palindrome $B$ of $W_{n}^{(k)}$ is called a maximal bordering palindrome if there is no longer bordering palindromic factor of $W_{n}^{(k)}$ with the same center position.

Lemma 21. Let $B_{j}$ be one of the bordering palindromes of type $j$ of $W_{n}^{(k)}$. Then

$$
c_{p}\left(B_{j}, W_{n}^{(k)}\right)=\left|W_{n-1}^{(k)}\right|+\cdots+\left|W_{j}^{(k)}\right|
$$

and $j$ is the center of $B_{j}$.

Proof. By Definition 11, we know that $B_{j}$ is a factor of $W=W_{j}^{(k)} W_{j-1}^{(k)} \cdots W_{n-k+1}^{(k)}$, and it contains the last digit of $W_{j}^{(k)}$, which is $j$. By Lemma $9,|W|_{j}=1$. Hence $\left|B_{j}\right|$ is an odd integer and $j$ is the center of $B_{j}$. Moreover $c_{p}\left(B_{j}, W_{n}^{(k)}\right)=\left|W_{n-1}^{(k)}\right|+\cdots+\left|W_{j}^{(k)}\right|$.

Lemma 22. Let $B_{j}$ be a bordering palindrome of type $j$ of $W_{n}^{(k)}$. Then $n \leqslant 2 k-3$ and $n-k+2 \leqslant j \leqslant k-1$.

Proof. Let $c=c_{p}\left(B_{j}, W_{n}^{(k)}\right)$ and $j^{\prime}=(j \bmod k)$. By Theorem 16 and Lemma 21, we have $B_{j}[c]=j$ and

$$
B_{j}[c-1]= \begin{cases}j-j^{\prime} & \text { if }\left(j^{\prime} \neq 0\right) \\ j-k+1 & \text { otherwise } .\end{cases}
$$

Using Definition 10 and Equation (6), we have $B_{j}[c+1]=0$. If $j^{\prime} \neq 0$, then by definition of $c$, we obtain $B_{j}[c+1]=B_{j}[c-1]=j-j^{\prime}$. Since $B_{j}[c+1]=0, j=j^{\prime}$, which shows that $0 \leqslant j \leqslant k-1$. Moreover, by Equation (6), $n-k+2 \leqslant j \leqslant n-1$. Hence $n-k+2 \leqslant j \leqslant k-1$, which shows that $n \leqslant 2 k-3$. If $j^{\prime}=0$, then $B_{j}[c-1]=j-k+1$ Using $B_{j}[c+1]=B_{j}[c-1]=j-k+1, B_{j}[c+1]=0$ and $j \geqslant n-k+2$, we have $j=k-1$ and $n \leqslant 2 k-3$, as desired.

Lemma 23. Let $k \leqslant n \leqslant 2 k-3$ and $n-k+2 \leqslant j \leqslant k-1$. Then the maximal bordering palindrome of type $j$ of $W_{n}^{(k)}$ is $B_{j}=\left(W_{j-1}^{(k)} W_{j-2}^{(k)} \cdots W_{n-k+1}^{(k)}\right)^{R} j\left(W_{j-1}^{(k)} W_{j-2}^{(k)} \cdots W_{n-k+1}^{(k)}\right)$. 
Proof. By Definition 11, $B_{j}$ is a palindromic factor of the following word

$$
W_{j}^{(k)} j^{-1} j W_{j-1}^{(k)} W_{j-2}^{(k)} \cdots W_{n-k+1}^{(k)},
$$

and by Lemma $21, j$ is the center of $B_{j}$. Using Lemma $22, j \leqslant k-1$ and $n-k+1 \leqslant j-1$. On the other hand by Lemma 4 , we have $W_{j-1}^{(k)} W_{j-2}^{(k)} \cdots W_{n-k+1}^{(k)} \triangleleft W_{j}^{(k)} j^{-1}$. Hence, using Lemma $18,\left(W_{j-1}^{(k)} W_{j-2}^{(k)} \cdots W_{n-k+1}^{(k)}\right)^{R} \triangleright W_{j}^{(k)} j^{-1}$. Therefore,

$$
B_{j}=\left(W_{j-1}^{(k)} W_{j-2}^{(k)} \cdots W_{n-k+1}^{(k)}\right)^{R} j\left(W_{j-1}^{(k)} W_{j-2}^{(k)} \cdots W_{n-k+1}^{(k)}\right) .
$$

Lemma 24. Let $n$ and $j$ be two integers with $k \leqslant n \leqslant 2 k-3$ and $n-k+2 \leqslant j \leqslant k-1$ and $B_{j}$ be a maximal bordering palindrome of type $j$ of $W_{n}^{(k)}$. Then $\left|B_{j}\right|=2\left(\left|W_{j}^{(k)}\right|-\right.$ $\left.\left|W_{n-k+1}^{(k)}\right|\right)+1$.

Proof. By Lemma 23, we have

$$
B_{j}=\left(W_{j-1}^{(k)} W_{j-2}^{(k)} \cdots W_{n-k+1}^{(k)}\right)^{R} j\left(W_{j-1}^{(k)} W_{j-2}^{(k)} \cdots W_{n-k+1}^{(k)}\right) .
$$

Using Equation (5),

$$
W_{j-1}^{(k)} W_{j-2}^{(k)} \cdots W_{n-k+1}^{(k)}=W_{j}^{(k)}\left(W_{n-k}^{(k)} \cdots W_{0}^{(k)} j\right)^{-1} .
$$

Hence,

$$
\begin{aligned}
\left|B_{j}\right| & =2\left(\left|W_{j}^{(k)}\right|-\left|W_{n-k}^{(k)} \cdots W_{0}^{(k)} j\right|\right)+1 \\
& =2\left(\left|W_{j}^{(k)}\right|-\left|W_{n-k+1}^{(k)}\right|\right)+1 .
\end{aligned}
$$

Lemma 25. Let $n \geqslant k$, then

$$
B^{(k)}(n, j)=\left\{\begin{array}{cl}
2^{j}-2^{n-k+1} & \text { if }(k \leqslant n \leqslant 2 k-3) \text { and }(n-k+2 \leqslant j \leqslant k-1), \\
0 & \text { otherwise. }
\end{array}\right.
$$

Proof. If $n \leqslant 2 k-3$ and $n-k+2 \leqslant j \leqslant k-1$ and $B_{j}$ be the maximal bordering palindrome of type $j$ of $W_{n}^{(k)}$. then by Lemmas 23 and 24 , we have

$$
\begin{aligned}
B^{(k)}(n, j) & =\frac{\left|B_{j}\right|-1}{2} \\
& =\left|W_{j}^{(k)}\right|-\left|W_{n-k+1}^{(k)}\right| \\
& =2^{j}-2^{n-k+1} .
\end{aligned}
$$

The last equality holds using Corollary 8. In other cases, by Lemma $22, B^{(k)}(n, j)=0$, as desired. 


\subsection{Straddling Palindromes of $W_{n}^{(k)}$}

In this subsection, we are going to count the number of straddling palindromes of $W_{n}^{(k)}$ when $n \geqslant k$. A straddling palindrome of $W_{n}^{(k)}$ is called a maximal straddling palindrome of $W_{n}^{(k)}$ if there is no longer straddling palindromic factor of $W_{n}^{(k)}$ with the same center position. Similarly, an $(A, B)$-maximal straddling palindrome, is an $(A, B)$-straddling palindrome which is a maximal straddling palindrome.

Lemma 26. If $S$ is a straddling palindrome of $W_{n}^{(k)}$, then $2 k-1 \leqslant n \leqslant 3 k-2$.

Proof. First we note that $\left(k \oplus W_{n-k}^{(k)}\right)$ starts with $k$ and $W_{n-k+1}^{(k)}$ ends with $n-k+1$. Hence, the sequence $(n-k+1) . k$ occurs in any straddling palindrome $P$ of $W_{n}^{(k)}$. Since $P$ is a palindrome, the sequence $B=k .(n-k+1)$ also occurs in $P$. Hence, by Lemma $13, B \in \mathcal{B}^{(k)}$. Since $n \geqslant k$, using Definition 12 , we provide $B \in \mathcal{B}_{1}^{(k)} \cup \mathcal{B}_{2}^{(k)}$. If $B \in \mathcal{B}_{1}^{(k)}$, then $B=k .(n-k+1)=(a . k) \oplus k i$, for some integers $a \neq 0$ and $i \geqslant 0$. Since $a \neq 0$, we conclude that $a=k$ and $i=0$. So, in this case $n-k+1=k$ and $n=2 k-1$.

If $B \in \mathcal{B}_{2}^{(k)}$, then $B=k \cdot(n-k+1)=(0 . b) \oplus k i$ for some $i \geqslant 0$ and $1 \leqslant b \leqslant k-1$. Hence, $i=1$ and $k+1 \leqslant n-k+1 \leqslant 2 k-1$. Therefore, $2 k \leqslant n \leqslant 3 k-2$, as desired.

Lemma 27. The only straddling palindrome of $W_{2 k-1}^{(k)}$ is $k . k$.

Proof. By Lemma 6, $W_{2 k-1}^{(k)}=W_{2 k-2}^{(k)} \cdots W_{k}^{(k)}\left(k \oplus W_{k-1}^{(k)}\right)$. It is clear that $k \triangleleft\left(k \oplus W_{k-1}^{(k)}\right)$ and using Theorem 16, $(1 . k) \triangleright W_{k}^{(k)}$.Hence, $k . k$ is a straddling palindrome of $W_{2 k-1}^{(k)}$. Now, we are going to show that there is no other straddling palindrome in $W_{2 k-1}^{(k)}$. Since there is no digit 1 in $\left(k \oplus W_{k-1}^{(k)}\right)$, every straddling palindrome of $W_{2 k-1}^{(k)}$ has the last digit of $W_{k}^{(k)}$ (i.e. $k$ ) as a prefix. By Corollary $14,0.0$ is not a factor of $W_{k-1}^{(k)}$ and hence $k . k$ is not a factor of $\left(k \oplus W_{k-1}^{(k)}\right)$. Therefore, $W_{2 k-1}^{(k)}$ could not have a straddling palindrome of length greater than 2 and $k . k$ is its only straddling palindrome.

Lemma 28. Let $2 k-1<n<3 k-2$ and let $W$ be an $(A, B)$-maximal straddling palindrome of $W_{n}^{(k)}$. Then $A \triangleright\left(k \oplus W_{n-2 k+1}^{(k)}\right)$ and hence $|A| \leqslant 2^{n-2 k+1}$.

Proof. Let $i=n-2 k$. Then, by Equation (6),

$$
\begin{aligned}
W_{2 k+i}^{(k)} & =W_{2 k+i-1}^{(k)} \cdots W_{k+i+1}^{(k)}\left(k \oplus W_{k+i}^{(k)}\right), \\
W_{k+i+1}^{(k)} & =W_{k+i}^{(k)} \cdots W_{i+2}^{(k)}\left(k \oplus W_{i+1}^{(k)}\right) .
\end{aligned}
$$

Hence,

$$
\begin{aligned}
W_{k+i+1}^{(k)}\left(k \oplus W_{k+i}^{(k)}\right) & \triangleright W_{2 k+i}^{(k)}, \\
W_{i+2}^{(k)}\left(k \oplus W_{i+1}^{(k)}\right) & \triangleright W_{k+i+1}^{(k)} .
\end{aligned}
$$

Therefore,

$$
W_{i+2}^{(k)}\left(k \oplus W_{i+1}^{(k)}\right)\left(k \oplus W_{k+i}^{(k)}\right) \triangleright W_{2 k+i}^{(k)}
$$


Let $W_{2 k+i}^{(k)}=V W_{i+2}^{(k)}\left(k \oplus W_{i+1}^{(k)}\right)\left(k \oplus W_{k+i}^{(k)}\right)$ and $\ell=\left|V W_{i+2}^{(k)}\right|$. By definition of the $(A, B)$ straddling palindrome of $W_{2 k+i}^{(k)}$ and using Equation (9), we conclude that $A \triangleright V W_{i+2}^{(k)}(k \oplus$ $\left.W_{i+1}^{(k)}\right)$. Let $A=W_{2 k+i}^{(k)}\left[j, j^{\prime}\right]$, where by definition of $A, j^{\prime}=\left|W_{2 k+i}^{(k)}\right|-\left|W_{k+i}^{(k)}\right|$. We claim that $j>\ell$. For the contrary, let $j \leqslant \ell$. The letter following $i+2$ in Equation (9) is $k$. Hence, $(i+2) k \prec W$ and so $k(i+2) \prec W$, but this is impossible by Corollary 15 , since $1<i+2<k$. Therefore, $A \triangleright\left(k \oplus W_{i+1}^{(k)}\right)$. Hence, $|A| \leqslant\left|W_{i+1}^{(k)}\right|$, so by Corollary 8 , $|A| \leqslant 2^{i+1}$.

The next lemma is useful to give an upper bound for the size of the word $B$ in any $(A, B)$-maximal straddling palindrome of $W_{n}^{(k)}, 2 k \leqslant n \leqslant 3 k-2$.

Lemma 29. Let $i \geqslant 0$ and $P$ be palindromic prefix of $W=(i+1) W_{k+i}^{(k)}$. Then the largest digit of $P$ is $i+1$.

Proof. Let $\ell$ be the largest digit of $P$ and for contrary suppose that $\ell>i+1$. Using Lemma 9, we have $\ell<i+k$. Hence, $\ell+1 \leqslant i+k$ which yields $W_{\ell+1}^{(k)} \triangleleft W_{i+k}^{(k)}$. We note that $\ell+1$, which is the last digit of $W_{\ell+1}^{(k)}$, does not appear in $P$. Hence,

$$
|P| \leqslant\left|W_{\ell+1}^{(k)}\right| \text {. }
$$

On the other hand $W_{\ell}^{(k)} \triangleleft W_{\ell+1}^{(k)}$ and since $i+1<\ell$ and using Lemma 9, we conclude that the first place that $\ell$ occurs in $W$ is the last digit of $W_{\ell}^{(k)}$. In other words if we let $m=\left|W_{\ell}^{(k)}\right|+1$, then $W[m]=\ell$ and for any integer $j<m$ we have $W[j]<\ell$. By our assumption $\ell \in \mathcal{A} l p h(P)$, therefore $c_{p}(P, W) \geqslant m=\left|W_{\ell}^{(k)}\right|+1$. Thus $|P| \geqslant 2\left|W_{\ell}^{(k)}\right|+1$ and using Equation (10), we have $|P| \leqslant\left|W_{\ell+1}^{(k)}\right|$. Hence, $2\left|W_{\ell}^{(k)}\right|+1 \leqslant\left|W_{\ell+1}^{(k)}\right|$ but this contradicts with Corollary 8. Hence our assumption is not true and $\ell \leqslant i+1$, as desired.

Lemma 30. Let $2 k-1<n<3 k-2$ and let $W$ be an $(A, B)$-maximal straddling palindrome of $W_{n}^{(k)}$. Then $B \triangleleft k \oplus\left(W_{n-2 k+2}^{(k)}(n-2 k+2)^{-1}\right)$ and hence $|B| \leqslant 2^{n-2 k+2}-1$.

Proof. Let $i=n-2 k$. Then, using Equation (6) we have

$$
W_{2 k+i}^{(k)}=W_{2 k+i-1}^{(k)} \cdots W_{k+i+1}^{(k)}\left(k \oplus W_{k+i}^{(k)}\right) .
$$

So we have

$$
B \triangleleft\left(k \oplus W_{k+i}^{(k)}\right) .
$$

By Lemma 28,

$$
A \triangleright\left(k \oplus W_{i+1}^{(k)}\right)
$$

Hence, $k+i+2 \notin \mathcal{A l p h}(A)$. We claim that $k+i+2 \notin \mathcal{A l p h}(B)$. For contrary suppose that $k+i+2 \in \mathcal{A l p h}(B)$. We note that $\left(k \oplus W_{i+2}^{(k)}\right) \triangleleft\left(k \oplus W_{k+i}^{(k)}\right)$. Now, let $m=\left|W_{i+2}^{(k)}\right|$, then by Lemma $9, B[m]=k+i+2$ and for all $m^{\prime}<m, B\left[m^{\prime}\right]<k+i+2$. On the other hand by Equation (11), $k+i+2 \notin \mathcal{A l p h}(A)$, hence $c_{p}(W, W) \geqslant|A|+m$. Therefore, $(k+i+1) B$ contains a palindrome prefix $P$ with $k+i+2 \in \mathcal{A l p h}(P)$, which is impossible by Lemma 29. Hence, $k+i+2 \notin \mathcal{A l p h}(B)$, which implies that $B \triangleleft k \oplus\left(W_{i+2}^{(k)}(i+2)^{-1}\right)$ and by Corollary $8,|B| \leqslant 2^{i+2}-1=2^{n-2 k+2}-1$. 
Now, we are ready to prove the following lemma.

Lemma 31. Let $2 k-1<n<3 k-2$. Then $W_{n}^{(k)}$ has exactly two maximal straddling palindromes, one of which is the $\left(k \oplus W_{n-2 k+1}^{(k)}, k \oplus\left(W_{n-2 k+1}^{(k)}(n-2 k+1)^{-1}\right)\right)$-straddling palindrome and the other is the $\left(k \oplus W_{n-2 k+1}^{(k)}, k \oplus\left(W_{n-2 k+1}^{(k)} W_{n-2 k+1}^{(k)}(n-2 k+1)^{-1}\right)\right)$ straddling palindrome.

Proof. Let $S=A B$ be an $(A, B)$-maximal straddling palindrome of $W_{n}^{(k)}$. Let $i=n-2 k$ and $W=k \oplus\left(W_{i+1}^{(k)} W_{i+2}^{(k)}(i+2)^{-1}\right)$. Then by Lemmas 28 and 30, we conclude that $S \prec W$. Moreover, by Equation (5) we have

$$
\begin{aligned}
W & =k \oplus\left(W_{i+1}^{(k)} W_{i+2}^{(k)}(i+2)^{-1}\right) \\
& =k \oplus\left(W_{i+1}^{(k)} W_{i+1}^{(k)} W_{i}^{(k)} \cdots W_{0}^{(k)}\right) \\
& =k \oplus\left(W_{i+1}^{(k)} W_{i+1}^{(k)} W_{i+1}^{(k)}(i+1)^{-1}\right) \\
& =k \oplus\left(W_{i+1}^{(k)}(i+1)^{-1}(i+1) W_{i+1}^{(k)}(i+1)^{-1}(i+1) W_{i+1}^{(k)}(i+1)^{-1}\right) .
\end{aligned}
$$

By the last equation and using Lemma 9, we conclude that $|W|_{k+i+1}=2$. Let $c_{1}$ and $c_{2}$ be two integers with $c_{1}<c_{2} \leqslant|W|$ such that $W\left[c_{1}\right]=W\left[c_{2}\right]=k+i+1$. Using Equation (6), we have

$$
\begin{aligned}
W_{2 k+i}^{(k)} & =W_{2 k+i-1}^{(k)} \cdots W_{k+i+2}^{(k)} W_{k+i+1}^{(k)}\left(k \oplus W_{k+i}^{(k)}\right) \\
& =W_{2 k+i-1}^{(k)} \cdots W_{k+i+2}^{(k)}\left(W_{k+i}^{(k)} \cdots W_{i+2}^{(k)}\left(k \oplus W_{i+1}^{(k)}\right)\right)\left(k \oplus W_{k+i}^{(k)}\right)
\end{aligned}
$$

$W$ starts by $\left(k \oplus W_{k+i}^{(k)}\right)$ in the previous expression. Thus, the first occurrence of $k+i+1$ in $W$ is the last letter of $\left(k \oplus W_{k+i}^{(k)}\right)$. Since $S$ is a straddling palindrome of $W_{2 k+i}^{(k)}$, the first occurrence of the digit has to be in $S$. Therefore, either $c_{p}(S, W)=c_{1}$ or $c_{p}(S, W)=\frac{c_{1}+c_{2}}{2}$. In other words, the center position of $S$ is either the corresponding position of the first occurrence of $k+i+1$, or is the position of the middle digit between the two occurrences of $k+i+1$ in $W$. So, we have the following two cases

- $S$ has only one digit $k+i+1$ : in this case we show that $A=k \oplus W_{i+1}^{(k)}$ and $B=$ $k \oplus\left(W_{i+1}^{(k)}(i+1)^{-1}\right)$. By Lemma 28 , it suffices to show that $k \oplus\left(W_{i+1}^{(k)} W_{i+1}^{(k)}(i+1)^{-1}\right)$ is a palindrome and this is true by Lemma 18 .

- $S$ has exactly two digits $k+i+1$ : in this case we show that $A=k \oplus W_{i+1}^{(k)}$ and $B=k \oplus\left(W_{i+1}^{(k)} W_{i+1}^{(k)}(i+1)^{-1}\right)$. By Lemma 18, $k \oplus\left(W_{i+1}^{(k)} W_{i+1}^{(k)} W_{i+1}^{(k)}(i+1)^{-1}\right)$ is a palindrome and using Lemmas 28 and 30 and Equation (12), $S=A B$ is a maximal straddling palindrome, as desired.

Lemma 32. Let $n=3 k-2$ and let $W$ be an $(A, B)$-maximal straddling palindrome of $W_{n}^{(k)}$. Then $A \triangleright\left(k \oplus W_{k-1}^{(k)}\right)$ and hence $|A| \leqslant 2^{k-1}$. 
Proof. By Lemma 6, we have

$$
\begin{aligned}
& W_{3 k-2}^{(k)}=W_{3 k-3}^{(k)} \cdots W_{2 k-1}^{(k)}\left(k \oplus W_{2 k-2}^{(k)}\right), \\
& W_{2 k-1}^{(k)}=W_{2 k-2}^{(k)} \cdots W_{k}^{(k)}\left(k \oplus W_{k-1}^{(k)}\right) .
\end{aligned}
$$

Hence, $W_{k}^{(k)}\left(k \oplus W_{k-1}^{(k)}\right)\left(k \oplus W_{2 k-2}^{(k)}\right) \triangleright W_{3 k-2}^{(k)}$. Since $1 k \triangleright W_{k}^{(k)}$ and by Corollary 15, $k 1 \nprec W_{3 k-2}^{(k)}$, we conclude that $|A| \leqslant\left|k\left(k \oplus W_{k-1}^{(k)}\right)\right|$. Hence, using Corollary 8, we have $|A| \leqslant 2^{k-1}+1$. Moreover, $k k \triangleleft k\left(k \oplus W_{k-1}^{(k)}\right)$, but $k k$ could not appear in $k \oplus\left(W_{k-1}^{(k)} W_{2 k-2}^{(k)}\right)$, because otherwise $00 \prec\left(W_{k-1}^{(k)} W_{2 k-2}^{(k)}\right)$ which is impossible by Corollary 14 . Therefore, $A \triangleright\left(k \oplus W_{k-1}^{(k)}\right)$ and using Corollary $8,|A| \leqslant 2^{k-1}$.

Lemma 33. Let $n=3 k-2$ and let $W$ be an $(A, B)$-maximal straddling palindrome of $W_{n}^{(k)}$. Then $B \triangleleft k \oplus\left(W_{k}^{(k)} k^{-1}\right)$, hence, $|B| \leqslant 2^{k}-1$.

Proof. Using Equation (6) we have

$$
\begin{aligned}
& W_{3 k-2}^{(k)}=W_{3 k-3}^{(k)} \cdots W_{2 k-1}^{(k)}\left(k \oplus W_{2 k-2}^{(k)}\right), \\
& W_{2 k-1}^{(k)}=W_{2 k-2}^{(k)} \cdots W_{k}^{(k)}\left(k \oplus W_{k-1}^{(k)}\right) .
\end{aligned}
$$

So we have

$$
B \triangleleft\left(k \oplus W_{2 k-2}^{(k)}\right) .
$$

By Lemma 32,

$$
A \triangleright\left(k \oplus W_{k-1}^{(k)}\right)
$$

Hence, $2 k \notin \mathcal{A} l p h(A)$. We claim that $2 k \notin \mathcal{A l p h}(B)$ as well. For contrary suppose that $2 k \in \mathcal{A l p h}(B)$. We note that $\left(k \oplus W_{k}^{(k)}\right) \triangleleft\left(k \oplus W_{2 k-2}^{(k)}\right)$. Now, let $m=\left|W_{k}^{(k)}\right|$, then by Lemma $9, B[m]=2 k$ and for all $m^{\prime}<m, B\left[m^{\prime}\right]<2 k$. On the other hand, $2 k \notin \mathcal{A l p h}(A)$, hence $c_{p}(W, W) \geqslant|A|+m$. Therefore, $(2 k-1) B$ contains a palindrome prefix $P$ with $2 k \in \mathcal{A l p h}(P)$, which is impossible by Lemma 29 . Hence, $2 k \notin \mathcal{A l p h}(B)$, which implies that $B \triangleleft k \oplus\left(W_{k}^{(k)}(k)^{-1}\right)$ and by Corollary $8|B| \leqslant 2^{k}-1$.

Lemma 34. Let $n=3 k-2$. Then $W_{n}^{(k)}$ has exactly two maximal straddling palindromes which are respectively the $\left(k \oplus W_{k-1}^{(k)}, k \oplus\left(W_{k-1}^{(k)}(k-1)^{-1}\right)\right)$-straddling palindrome and the $\left(k \oplus\left(0^{-1} W_{k-1}^{(k)}\right), k \oplus\left(W_{k-1}^{(k)} W_{k-1}^{(k)}(0(k-1))^{-1}\right)\right)$-straddling palindrome in $W_{n}^{(k)}$.

Proof. Let $W$ be an $(A, B)$-maximal straddling palindrome of $W_{3 k-2}^{(k)}$. Using Equation (6) we have

$$
\begin{aligned}
k \oplus\left(W_{k}^{(k)} k^{-1}\right) & =k \oplus\left(W_{k-1}^{(k)} W_{k-2}^{(k)} \cdots W_{1}^{(k)} k \cdot k^{-1}\right) \\
& =k \oplus\left(W_{k-1}^{(k)} W_{k-2}^{(k)} \cdots W_{1}^{(k)}\right) \\
& =k \oplus\left(W_{k-1}^{(k)} W_{k-1}^{(k)}(0(k-1))^{-1}\right) .
\end{aligned}
$$


Therefore, using Lemmas 33 and 34 every $(A, B)$-straddling palindrome $S$ of $W_{3 k-2}^{(k)}$, we have $S=A B \prec W$, where

$$
\begin{aligned}
W & =k \oplus\left(W_{k-1}^{(k)} W_{k-1}^{(k)} W_{k-1}^{(k)}(0(k-1))^{-1}\right. \\
& =k \oplus\left(W_{k-1}^{(k)}(k-1)^{-1}(k-1) W_{k-1}^{(k)}(k-1)^{-1}(k-1) W_{k-1}^{(k)}(0(k-1))^{-1}\right) .
\end{aligned}
$$

By Lemma 9 and Equation (14) the digit $2 k-1$ occurs twice in $W$. Let $c_{1}$ and $c_{2}$ be two integers with $c_{1}<c_{2} \leqslant|W|$ such that $W\left[c_{1}\right]=W\left[c_{2}\right]=2 k-1$. Since $S$ is a straddling palindrome of $W_{3 k-2}^{(k)}, S$ should contain the first occurrence of $2 k-1$ in $W$. Therefore, either we have $c_{p}(S, W)=c_{1}$ or $c_{p}(S, W)=\frac{c_{1}+c_{2}}{2}$. In other words the center position of $S$ is either the position of the first occurrence of $2 k-1$ in $W$ or exactly the position of the digit in the middle of the two occurrences of $2 k-1$ in $W$. So, we have the following two cases

- $|S|_{2 k-1}=1$ : In this case we show that $A=k \oplus W_{k-1}^{(k)}$ and $B=k \oplus\left(W_{k-1}^{(k)}(k-1)^{-1}\right)$. By Lemma 32, it suffices to show that $k \oplus\left(W_{k-1}^{(k)} W_{k-1}^{(k)}(k-1)^{-1}\right)$ is a palindrome and this is true by Lemma 18 .

- $|S|_{2 k-1}=2$ : In this case we show that $A=k \oplus\left(0^{-1} W_{k-1}^{(k)}\right)$ and $B=k \oplus$ $\left(W_{k-1}^{(k)} W_{k-1}^{(k)}(0(k-1))^{-1}\right)$. By Lemma $18, k \oplus\left(0^{-1} W_{k-1}^{(k)} W_{k-1}^{(k)} W_{k-1}^{(k)}(0(k-1))^{-1}\right)$ is a palindrome and using Lemmas 32 and $33, S=A B$ is a maximal straddling palindrome as desired.

Theorem 35. Let $S^{(k)}(n)$ be the number of straddling palindromes of $W_{n}^{(k)}$. Then

$$
S^{(k)}(n)= \begin{cases}2^{n-2 k+2}-1 & \text { if } 2 k-1 \leqslant n<3 k-2, \\ 2^{k}-2 & \text { if } n=3 k-2, \\ 0 & \text { otherwise. }\end{cases}
$$

Proof. If $n=2 k-1$, then by Lemma 27, the only straddling palindrome of $W_{n}^{(k)}$ is $k k$. Hence, $S^{(k)}(2 k-1)=1$.

If $2 k \leqslant n \leqslant 3 k-3$, then by Lemma 31, the only maximal straddling palindromes of $W_{n}^{(k)}$ are $S_{1}=\left(k \oplus W_{i+1}^{(k)}\right)\left(k \oplus\left(W_{i+1}^{(k)}(i+1)^{-1}\right)\right.$ and $S_{2}=\left(k \oplus W_{i+1}^{(k)}\right)\left(k \oplus\left(W_{i+1}^{(k)} W_{i+1}^{(k)}(i+1)^{-1}\right)\right)$, where $i=n-2 k$. According to the proof of Lemma 31, the center of $S_{1}$ is $k+i+1$ and $c_{p}\left(S_{1}, S_{1}\right)=\left|W_{i+1}^{(k)}\right|$. Therefore, the number of straddling palindromes with the same center position as $S_{1}$, is $\left|W_{i+1}^{(k)}\right|-1$, which equals to $2^{i+1}-1=2^{n-2 k+1}-1$, using Corollary 8. Now, again by the proof of Lemma $31, c_{p}\left(S_{2}, S_{2}\right)>\left|W_{i+1}^{(k)}\right|$. Hence, by Corollary 8 , the number of straddling palindromes with the same center position as $S_{2}$, is $\left|W_{i+1}^{(k)}\right|=2^{i+1}=2^{n-2 k+1}$. Therefore, for $2 k \leqslant n \leqslant 3 k-3, S^{(k)}(n)=2^{n-2 k+1}+2^{n-2 k+1}-1=2^{n-2 k+2}-1$.

If $n=3 k-2$, then by Lemma 34, the only maximal straddling palindromes of $W_{n}^{(k)}$ are $S_{1}=k \oplus W_{k-1}^{(k)} k \oplus\left(W_{k-1}^{(k)}(k-1)^{-1}\right)$ and $S_{2}=k \oplus\left(0^{-1} W_{k-1}^{(k)}\right) k \oplus\left(W_{k-1}^{(k)} W_{k-1}^{(k)}(0(k-1))^{-1}\right)$. According to the proof of Lemma $34, c_{p}\left(S_{1}, S_{1}\right)=\left|W_{k-1}^{(k)}\right|$. Therefore, the number of straddling palindromes with the same center position as $S_{1}$ is $\left|W_{k-1}^{(k)}\right|-1$, which equals to 
$2^{k-1}-1$ by Corollary 8 . Now, again by the proof of Lemma $34, c_{p}\left(S_{2}, S_{2}\right)>\left|W_{k-1}^{(k)}\right|$. Hence, using Corollary 8, the number of straddling palindrome with the same center position as $S_{2}$, is $\left|0^{-1} W_{k-1}^{(k)}\right|=2^{k-1}-1$. Therefore, $S^{(k)}(3 k-2)=2^{k}-2$.

Finally, $S^{(k)}(n)=0$, when $n<2 k-1$ or $n>3 k-2$, using by Lemma 26 .

\subsection{Proof of Theorem 17}

Theorem 12. Let $k>2$ and $n \geqslant 0$ be given integers. Then the following holds

(i) If $0 \leqslant n \leqslant k-1$, then $P^{(k)}(n)=n 2^{n-1}+1$,

(ii) If $n \geqslant k$, then $P^{(k)}(n)=\sum_{i=n-k}^{n-1} P^{(k)}(i)+\alpha^{(k)}(n)$, where

$$
\alpha^{(k)}(n)= \begin{cases}2^{k}-k 2^{n-k+2}+n 2^{n-k+1} & \text { if } k \leqslant n \leqslant 2 k-3, \\ 0 & \text { if } n=2 k-2, \\ 2^{n-2 k+2}-1 & \text { if } 2 k-1 \leqslant n \leqslant 3 k-3, \\ 2^{k}-2 & \text { if } n=3 k-2, \\ 0 & \text { if } n>3 k-2 .\end{cases}
$$

Proof. (i) This part is true according to Theorem 20.

(ii) To prove this part by Equation (7) we have

$$
P^{(k)}(n)=\sum_{i=n-k}^{n-1} P^{(k)}(i)+\sum_{j=n-k+2}^{n-1} B^{(k)}(n, j)+S^{(k)}(n) .
$$

Therefore,

$$
P^{(k)}(n)=\sum_{i=n-k}^{n-1} P^{(k)}(i)+\alpha^{(k)}(n),
$$

where

$$
\alpha^{(k)}(n)=\sum_{j=n-k+2}^{n-1} B^{(k)}(n, j)+S^{(k)}(n) .
$$

By Theorem 35, when $k \leqslant n \leqslant 2 k-3, S^{(k)}(n)=0$. Therefore, $\alpha^{(k)}(n)=$ $\sum_{j=n-k+2}^{n-1} B^{(k)}(n, j)$, when $k \leqslant n \leqslant 2 k-3$. Now, by Lemmas 22 and 25 we have

$$
\begin{aligned}
\alpha^{(k)}(n) & =\sum_{j=n-k+2}^{n-1} B^{(k)}(n, j) \\
& =\sum_{j=n-k+2}^{k-1} B^{(k)}(n, j) \text { by Lemma } 22 \\
& =\sum_{j=n-k+2}^{k-1}\left(2^{j}-2^{n-k+1}\right) \\
& =2^{k}-k 2^{n-k+2}+n 2^{n-k+1} .
\end{aligned}
$$

If $2 k-1 \leqslant n \leqslant 3 k-2$, then by Lemma 25 and Equation $(15), \alpha^{(k)}(n)=S^{(k)}(n)$. Hence using Theorem 35, we have

$$
\alpha^{(k)}(n)= \begin{cases}2^{n-2 k+2}-1 & \text { if } 2 k-1 \leqslant n \leqslant 3 k-3, \\ 2^{k}-2 & \text { if } n=3 k-2 .\end{cases}
$$

Finally, using Theorem 35 and Lemma 25, $\alpha^{(k)}(n)=0$, if either $n=2 k-2$ or $n>3 k-2$. 


\subsection{Examples}

In the following example in the case $k=4$ for some different values of $n$ we give all of the maximal straddling palindromes and the maximal bordering palindromes of $W_{n}^{(k)}$, if there exists any.

Example 36. Let $k=4$. Then by Lemma 23, the word $W_{4}^{(4)}$ has two maximal bordering palindromes $B_{2}=\left(W_{1}^{(4)}\right)^{R} 2\left(W_{1}^{(4)}\right)$ and $B_{3}=\left(W_{2}^{(4)} W_{1}^{(4)}\right)^{R} 3\left(W_{2}^{(4)} W_{1}^{(4)}\right)$, which are shown below. We also notice that by Theorem $35, W_{4}^{(4)}$ has no straddling palindrome.

$$
\begin{aligned}
& W_{4}^{(4)}=01020103.0102 .01 .4 \\
& W_{4}^{(4)}=01020103.0102 .01 .4 .
\end{aligned}
$$

In the case $n=5=2 k-3$, by Lemma 23 , the word $W_{5}^{(4)}$ has a maximal bordering palindrome $B_{3}=\left(W_{2}^{(4)}\right)^{R} 3\left(W_{2}^{(4)}\right)$, which is shown bellow. We also notice that by Theorem $35, W_{5}^{(4)}$ has no straddling palindrome.

$$
W_{5}^{(4)}=010201030102014.01020103 .0102 .45
$$

In the case $n=6$, by Theorem 35 and Lemma 25, $W_{6}^{(4)}$ contains neither a straddling palindrome nor a bordering palindrome.

$$
W_{6}^{(4)}=01020103010201401020103010245.010201030102014 .01020103 .4546
$$

Example 37. Let $k=4$. Again by the same reasoning as the previous example the word $W_{7}^{(4)}$ has one straddling palindrome and no bordering palindrome.

$W_{7}^{(4)}=01020103010201401020103010245010201030102014010201034546.010201030102014$ 01020103010245.010201030102014 .45464547

By Lemma $25, W_{9}^{(4)}$ has no bordering palindrome and by Lemma 31 it has two maximal straddling palindromes which are given by $\left(4 \oplus W_{2}^{(4)}, 4 \oplus\left(W_{2}^{(4)} 2^{-1}\right)\right)$ and $\left(4 \oplus W_{2}^{(4)}, 4 \oplus\right.$ $\left.\left(W_{2}^{(4)} W_{2}^{(4)} 2^{-1}\right)\right)$ as shown below.

$W_{9}^{(4)}=01020103010201401020103010245010201030102014010201034546010201030102014$ 01020103010245010201030102014454645470102010301020140102010301024501020 103010201401020103454601020103010201401020103010245454645474546458.0102 01030102014010201030102450102010301020140102010345460102010301020140102 010301024501020103010201445464547.0102010301020140102010301024501020103 0102014010201034546.45464547454645845464547454689

$W_{9}^{(4)}=01020103010201401020103010245010201030102014010201034546010201030102014$ 
01020103010245010201030102014454645470102010301020140102010301024501020

103010201401020103454601020103010201401020103010245454645474546458.0102

01030102014010201030102450102010301020140102010345460102010301020140102

010301024501020103010201445464547.0102010301020140102010301024501020103

0102014010201034546.45464547454645845464547454689 .

\section{Palindrome Structure}

In this section, based on finding the structure of all palindromic factors of $W^{(k)}$, we compute its palindrome complexity, $\operatorname{pal}_{W^{(k)}}(n)$. We recall that for a fixed word $U \in \mathcal{A}^{\infty}$, $\operatorname{pal}_{U}(n)$ is the number of palindromic factors of length $n$ of $U$. Hence, $\operatorname{pal}_{U}(n)$ is a function from $\mathbb{N}$ to $\mathbb{N} \cup\{\infty\}$.

Definition 38. For a set $P$ of palindromic words, we define

$$
\begin{array}{r}
\mathcal{C P a l}(P)=\left\{W \in \mathcal{A}^{*} \mid \text { There exist } U \in P \text { and integers } i, j\right. \text { with } \\
1 \leqslant i \leqslant j \leqslant|U|, \quad i+j=|U|+1 \text { and } W=U[i, j]\} .
\end{array}
$$

Remark 39. It is obvious from Definition 38 that any element of $\mathcal{C P}$ al $(P)$ is a palindromic factor of some word of $P$, but there may exist other palindromic factors of words of $P$ which do not belong to $\mathcal{C P} a l(P)$ as is seen from the following example.

Example 40. Let $P=\{1213121,33433\}$. Then by Definition 38,

$$
\mathcal{C P a l}(P)=\{1213121,21312,131,3,33433,343,4\}
$$

Note that the words $1,2,33$ and 121 are palindromic factors of some words of $P$ but they are not elements of $\mathcal{C} \mathcal{P} a l(P)$.

Lemma 41. Let $U \in \mathcal{A}^{*}$ and $P \subseteq \mathcal{P}$ al $(U)$. Then $\mathcal{C P} a l(P) \subseteq \mathcal{P} a l(U)$.

Proof. Since any element of $P$ is a palindrome, by Definition 38, any element $W \in$ $\mathcal{C P a l}(P)$ is also a palindromic factor of $U$, whence the result follows.

Lemma 42. For any integer $k>2$ we have

$$
\mathcal{P} a l\left(W^{(k)}\right)=\bigcup_{i \geqslant 0}\left(k i \oplus\left(\bigcup_{j=1}^{3 k-2} \mathcal{P} a l\left(W_{j}^{(k)}\right)\right)\right) .
$$

Proof. Let $1 \leqslant j \leqslant 3 k-2$. By Equation (6), we have $\left(k \oplus W_{j}^{(k)}\right) \triangleright W_{j+k}^{(k)}$. Hence, using induction on $i$, for every nonnegative integer $i$ we provide

$$
\left(k i \oplus W_{j}^{(k)}\right) \triangleright W_{j+k i}^{(k)} .
$$


Now, since $W_{j+k i}^{(k)} \prec W^{(k)}$ we conclude that

$$
\bigcup_{i \geqslant 0}\left(k i \oplus\left(\bigcup_{j=1}^{3 k-2} \mathcal{P} a l\left(W_{j}^{(k)}\right)\right)\right) \subseteq \mathcal{P} a l\left(W^{(k)}\right) .
$$

To complete the proof, we need to show that

$$
\mathcal{P} a l\left(W_{n}^{(k)}\right) \subseteq \bigcup_{i \geqslant 0}\left(k i \oplus\left(\bigcup_{j=1}^{3 k-2} \mathcal{P} a l\left(W_{j}^{(k)}\right)\right)\right)
$$

holds for all integers $n$. But since $m<n$ implies that $W_{m}^{(k)} \prec W_{n}^{(k)}$ and $\mathcal{P} a l\left(W_{m}^{(k)}\right) \subset$ $\mathcal{P} a l\left(W_{n}^{(k)}\right)$, it suffices to prove that Equation (17) holds from a point on, say that it holds for all $n>3 k-3$. To prove this, we use strong induction on $n$. The basis step $n=3 k-2$ is obviously true because $\mathcal{P} a l\left(W_{3 k-2}^{(k)}\right)$ appears in the right side of Equation (17). For the inductive step, let $n>3 k-2$ and assume that Equation (17) holds for all integers $j$, $3 k-2 \leqslant j<n$. Since $3 k-2<n$, using Lemma 25 and Theorem $35, W_{n}^{(k)}$ has neither a straddling palindrome nor a bordering palindrome. Hence, by Equation (6), we have

$$
\mathcal{P} a l\left(W_{n}^{(k)}\right)=\bigcup_{j=n-k+1}^{n-1} \mathcal{P} a l\left(W_{j}^{(k)}\right) \bigcup\left(k \oplus\left(\mathcal{P} a l\left(W_{n-k}^{(k)}\right)\right)\right) .
$$

By the induction hypothesis we have

$$
\bigcup_{j=n-k+1}^{n-1} \mathcal{P} a l\left(W_{j}^{(k)}\right) \bigcup\left(k \oplus\left(\mathcal{P} a l\left(W_{n-k}^{(k)}\right)\right)\right) \subseteq \bigcup_{i \geqslant 0}\left(k i \oplus\left(\bigcup_{j=1}^{3 k-2} \mathcal{P} a l\left(W_{j}^{(k)}\right)\right)\right) .
$$

Therefore, Equation (17) holds for $j=n$, as desired.

To present the next results we need the following definition.

Definition 43. Let $k>2$. We define the following sets of words:

$$
\begin{aligned}
\mathcal{P}_{1}^{(k)}:= & \left\{k i \oplus\left(W_{n}^{(k)} n^{-1}\right): 1 \leqslant n \leqslant k-1, i \geqslant 0\right\} \\
\mathcal{P}_{2}^{(k)}:= & \left\{k i \oplus\left(\left(W_{j-1}^{(k)} W_{j-2}^{(k)} \cdots W_{n-k+1}^{(k)}\right)^{R} j\left(W_{j-1}^{(k)} W_{j-2}^{(k)} \cdots W_{n-k+1}^{(k)}\right)\right):\right. \\
& k \leqslant n \leqslant 2 k-3, n-k+2 \leqslant j \leqslant k-1, \quad i \geqslant 0\}, \\
\mathcal{P}_{3}^{(k)}:= & \left\{k i \oplus\left(W_{n-2 k+1}^{(k)} W_{n-2 k+1}^{(k)}(n-2 k+1)^{-1}\right), k i \oplus\left(W_{n-2 k+1}^{(k)} W_{n-2 k+1}^{(k)} W_{n-2 k+1}^{(k)}(n-2 k+1)^{-1}\right):\right. \\
& 2 k \leqslant n \leqslant 3 k-3, \quad i \geqslant 1\}, \\
\mathcal{P}_{4}^{(k)}:= & \left\{k i \oplus\left(W_{k-1}^{(k)} W_{k-1}^{(k)}(k-1)^{-1}\right), k i \oplus\left(0^{-1} W_{k-1}^{(k)} W_{k-1}^{(k)} W_{k-1}^{(k)}(0(k-1))^{-1}\right), k i \oplus(00): i \geqslant 1\right\} .
\end{aligned}
$$

Lemma 44. Let $k>2$. Then $\mathbb{N} \subseteq \mathcal{C P}$ al $\left(P_{1}^{(k)} \cup P_{2}^{(k)}\right)$.

Proof. Let $m \in \mathbb{N}$ and $m=(j \bmod k)$. If $j=0$ or $j=1$, then by Definition 43 , we have $0,010 \in \mathcal{P}_{1}^{(k)}$ and hence $m \in \mathcal{C} \mathcal{P} a l\left(\mathcal{P}_{1}^{(k)}\right)$. Otherwise $2 \leqslant j \leqslant k-1$ and using Lemma 23, for every integer $n$ satisfying $k \leqslant n \leqslant k+j-2$ we have

$$
\left(W_{j-1}^{(k)} W_{j-2}^{(k)} \cdots W_{n-k+1}^{(k)}\right)^{R} j\left(W_{j-1}^{(k)} W_{j-2}^{(k)} \cdots W_{n-k+1}^{(k)}\right) \in \mathcal{P} a l\left(W_{n}^{(k)}\right) .
$$

Hence, $j \in \mathcal{C} \mathcal{P} a l\left(\mathcal{P}_{2}^{(k)}\right)$ and by Definition $43, m \in \mathcal{C P}$ al $\left(\mathcal{P}_{2}^{(k)}\right)$ as well. 
Lemma 45. Let $k>2$. Then $\bigcup_{i=1}^{4} \mathcal{P}_{i}^{(k)} \subseteq \mathcal{P} a l\left(W^{(k)}\right)$.

Proof. Using Lemma 18,

$$
\left\{W_{n}^{(k)} n^{-1}: 1 \leqslant n \leqslant k-1\right\} \subseteq \mathcal{P} a l\left(W^{(k)}\right) .
$$

By Lemma 23, the set

$$
\begin{aligned}
& \left\{\left(W_{j-1}^{(k)} W_{j-2}^{(k)} \cdots W_{n-k+1}^{(k)}\right)^{R} j\left(W_{j-1}^{(k)} W_{j-2}^{(k)} \cdots W_{n-k+1}^{(k)}\right):\right. \\
& k \leqslant n \leqslant 2 k-3, \quad n-k+2 \leqslant j \leqslant k-1\} \subseteq \mathcal{P} a l\left(W^{(k)}\right) .
\end{aligned}
$$

By Lemma 31,

$$
\begin{aligned}
& \left\{k \oplus\left(W_{n-2 k+1}^{(k)} W_{n-2 k+1}^{(k)}(n-2 k+1)^{-1}\right), k \oplus\left(W_{n-2 k+1}^{(k)} W_{n-2 k+1}^{(k)} W_{n-2 k+1}^{(k)}(n-2 k+1)^{-1}\right):\right. \\
& 2 k \leqslant n \leqslant 3 k-3\} \subseteq \mathcal{P} a l\left(W^{(k)}\right) .
\end{aligned}
$$

Finally, by Lemmas 27 and 34,

$$
\left\{k \oplus\left(W_{k-1}^{(k)} W_{k-1}^{(k)}(k-1)^{-1}\right), k \oplus\left(0^{-1} W_{k-1}^{(k)} W_{k-1}^{(k)} W_{k-1}^{(k)}(0(k-1))^{-1}\right), k . k\right\} \subseteq \mathcal{P} a l\left(W^{(k)}\right) .
$$

On the other hand, using Equation (6), we conclude that if $P \in \mathcal{P} a l\left(W^{(k)}\right)$, then for every integer $i \geqslant 0$, ki $\oplus P \in \mathcal{P} a l\left(W^{(k)}\right)$. Therefore, using Equations (18)-(21), we conclude.

The following six lemmas give the structure of the palindromes of $W_{n}^{(k)}$, when $n \leqslant$ $3 k-2$.

Lemma 46. Let $k>2,1 \leqslant n<k$ and $P$ be a maximal palindromic factor of $W_{n}^{(k)}$ of length at least 2 . Then $P \in \mathcal{P}_{1}^{(k)}$.

Proof. By Lemma 18, $W_{n}^{(k)} n^{-1}=W_{n-1}^{(k)}(n-1)^{-1}(n-1) W_{n-1}^{(k)}(n-1)^{-1}$ is a maximal palindrome with center $n-1$. Therefore, it is easy to see that the maximal palindromes appearing in $W_{n}^{(k)}$ are either equal to $W_{n}^{(k)} n^{-1}$ or are a maximal palindrome of $W_{n-1}^{(k)}(n-$ $1)^{-1}$. Hence, using induction we can see that the set of all maximal palindromes of $W_{n}^{(k)}$ is $\left\{W_{i}^{(k)} i^{-1}: 2 \leqslant i \leqslant n\right\} \subset \mathcal{P}_{1}^{(k)}$.

Lemma 47. Let $2<k \leqslant n \leqslant 2 k-3$ and $P$ be a maximal palindromic factor of $W_{n}^{(k)}$ of length at least 2 , then $P \in \mathcal{P}_{1}^{(k)} \cup \mathcal{P}_{2}^{(k)}$.

Proof. We prove the result using induction on $n$. For the basis step let $n=k$ and $P \in \mathcal{P}_{2}^{(k)} \backslash \mathcal{P}_{1}^{(k)}$ be a maximal palindromic factor of $W_{n}^{(k)}$. Since $P \notin \mathcal{P}_{1}^{(k)}$, using Lemma 46, we conclude that $P$ is not an included palindromic factor of $W_{n}^{(k)}$. On the other hand by Theorem 35, $P$ is not a straddling factor of $W_{n}^{(k)}$. Hence, $P$ is a maximal bordering factor of $W_{n}^{(k)}$. Now, using Lemma 23, we provide $P \in \mathcal{P}_{2}^{(k)}$. 
For the inductive step, let $k<n \leqslant 3 k-3$ and suppose that the result is true for all $j, k \leqslant j<n$. Let $P$ be a maximal palindromic factor of $W_{n}^{(k)}$. Then by Theorem 35, either $P$ is an included maximal palindromic factor of $W_{n}^{(k)}$ or it is a bordering maximal palindromic factor of $W_{n}^{(k)}$. In the former case, using Lemma 46 and induction hypothesis we conclude that $P \in \mathcal{P}_{1}^{(k)} \cup \mathcal{P}_{2}^{(k)}$. In the latter case, by Lemma 23 we have $P \in \mathcal{P}_{2}^{(k)}$, as desired.

Lemma 48. Let $k>2$ and $n=2 k-2$ and $P$ be a maximal palindromic factor of $W_{n}^{(k)}$ of length at least 2 . Then $P \in \mathcal{P}_{1}^{(k)} \cup \mathcal{P}_{2}^{(k)}$.

Proof. By Lemma 25 and Theorem 35, $W_{n}^{(k)}$ has neither a straddling palindrome nor a bordering palindrome. Hence, using Equation (6), Lemmas 46 and 47, $\mathcal{P a l}\left(W_{n}^{(k)}\right) \subseteq$ $\left(\mathcal{P}_{1}^{(k)} \cup \mathcal{P}_{2}^{(k)}\right)$, as desired.

Lemma 49. Let $k>2$ and $n=2 k-1$ and $P$ be a maximal palindromic factor of $W_{n}^{(k)}$ of length at least 2 . Then either $P=k$. $k$ or $P \in \mathcal{P}_{1}^{(k)} \cup \mathcal{P}_{2}^{(k)}$.

Proof. Let $P \notin \mathcal{P}_{1}^{(k)} \cup \mathcal{P}_{2}^{(k)}$. Then by Lemmas $46-48, P$ is not a maximal palindromic factor of any $W_{j}^{(k)}, j<n$. Hence, $P$ is either a straddling palindrome or a bordering palindrome. By Lemmas 22 and $27, P$ is straddling and $P=k . k$.

Lemma 50. Let $k>2$ and $2 k \leqslant n \leqslant 3 k-3$ and $P$ be a maximal palindromic factor of $W_{n}^{(k)}$ of length at least 2 . Then $P \in\left(\mathcal{P}_{1}^{(k)} \cup \mathcal{P}_{2}^{(k)} \cup \mathcal{P}_{3}^{(k)} \cup\{k i . k i: i \geqslant 1\}\right)$.

Proof. We use induction on $n$. First, let $n=2 k$ and let $P$ be a maximal palindromic factor of $W_{2 k}^{(k)}$. Then by Lemma $25, P$ is not a bordering factor, hence, it is either an included palindromic factor or a straddling palindromic factor of $W_{2 k}^{(k)}$. In the former case, by Lemmas 46-49, $P \in\left(\mathcal{P}_{1}^{(k)} \cup \mathcal{P}_{2}^{(k)} \cup\{k i . k i: i \geqslant 1\}\right)$, while in the latter case, by Lemma $31, P \in \mathcal{P}_{3}^{(k)}$. This terminates the basis step of the induction.

For the inductive step, consider any integer $n, 2 k<n \leqslant 3 k-3$, and assume that the lemma holds for all integers $j, 2 k \leqslant j<n$. To conclude the validity of lemma for $n$, note that a maximal palindromic factor $P$ of $W_{n}^{(k)}$ is either an included or a straddling palindrome, by Lemma 25. In the former case, by induction hypothesis, $P \in\left(\mathcal{P}_{1}^{(k)} \cup \mathcal{P}_{2}^{(k)} \cup\right.$ $\mathcal{P}_{3}^{(k)} \cup\{k i . k i: i \geqslant 1\}$ ), while in the latter case, by Lemma 31, $P \in \mathcal{P}_{3}^{(k)}$. Hence, we are done.

Lemma 51. Let $k>2, n=3 k-2$ and $P$ be a maximal palindromic factor of $W_{n}^{(k)}$ of length at least 2. Then $P \in \bigcup_{i=1}^{4} \mathcal{P}_{i}^{(k)}$.

Proof. Let $P \notin\left(\mathcal{P}_{1}^{(k)} \cup \mathcal{P}_{2}^{(k)} \cup \mathcal{P}_{3}^{(k)} \cup\{k i . k i: i \geqslant 1\}\right)$. Thus, by Lemmas 46-50, $P$ is not a maximal palindromic factor of any $W_{j}^{(k)}, j<n$. Hence, $P$ is either a straddling palindrome or a bordering palindrome. By Lemma $25, W_{n}^{(k)}$ has no bordering palindrome and hence it is a maximal straddling palindrome of $W_{n}^{(k)}$. By Lemma 34, we conclude that $P \in \mathcal{P}_{4}^{(k)}$. 
Lemma 52. Let $k>2$ and let $P$ be a maximal palindromic factor of $W^{(k)}$ of length at least 2. Then $P \in \bigcup_{i=1}^{4} \mathcal{P}_{i}^{(k)}$.

Proof. By Lemma 42, there exist $i \geqslant 0$ and $1 \leqslant j \leqslant 3 k-2$ such that $P^{\prime}$ is a maximal palindrome of $W_{j}^{(k)}$ and $P=k i \oplus P^{\prime}$. Now, using Lemmas 46-51, $P^{\prime} \in \bigcup_{i=1}^{4} \mathcal{P}_{i}^{(k)}$. Since $k i \oplus\left(\bigcup_{i=1}^{4} \mathcal{P}_{i}^{(k)}\right)=\bigcup_{i=1}^{4} \mathcal{P}_{i}^{(k)}$, we have $P \in \bigcup_{i=1}^{4} \mathcal{P}_{i}^{(k)}$.

Theorem 53. For any integer $k>2$ we have

$$
\mathcal{P} a l\left(W^{(k)}\right)=\mathcal{C P} \text { al }\left(\bigcup_{i=1}^{4} \mathcal{P}_{i}^{(k)}\right)
$$

Proof. First we prove that the left side of Equation (22) is a subset of its right side. For this, consider any element $P \in \mathcal{P} a l\left(W^{(k)}\right)$. If $|P|=1$, then by Lemma 44, $P \in$ $\mathcal{C P} a l\left(\bigcup_{i=1}^{4} \mathcal{P}_{i}^{(k)}\right)$. Otherwise, consider a maximal palindromic factor factor $U$ of $W^{(k)}$ such that $P \in \mathcal{C P}$ al $(\{U\})$. By Lemma 52, $U \in \bigcup_{i=1}^{4} \mathcal{P}_{i}^{(k)}$. therefore, using Lemma 41, $P \in$ $\mathcal{C P} a l\left(\bigcup_{i=1}^{4} \mathcal{P}_{i}^{(k)}\right)$ as required.

To prove that the right side of Equation (22) is a subset of its left side, note that by Lemma 45, $\bigcup_{i=1}^{4} \mathcal{P}_{i}^{(k)} \subseteq \mathcal{P} a l\left(W^{(k)}\right)$. Thus using Lemma 41, $\mathcal{C P} a l\left(\bigcup_{i=1}^{4} \mathcal{P}_{i}^{(k)}\right) \subseteq \mathcal{P} a l\left(W^{(k)}\right)$

Example 54. Consider the word $W^{(3)}$. Then by Definition 43 , the sets $\mathcal{P}_{1}^{(3)}, \mathcal{P}_{2}^{(3)}, \mathcal{P}_{3}^{(3)}, \mathcal{P}_{4}^{(3)}$ are as follows:

$$
\begin{aligned}
& \mathcal{P}_{1}^{(3)}=\{3 i, 3 i \oplus(010): i \geqslant 0\} \\
& \mathcal{P}_{2}^{(3)}=\{3 i \oplus(10201): i \geqslant 0\} \\
& \mathcal{P}_{3}^{(3)}=\{3 i \oplus(010), 3 i \oplus(01010): i \geqslant 1\}, \\
& \mathcal{P}_{4}^{(3)}=\{3 i \oplus(0102010), 3 i \oplus(102010201), 3 i \oplus(00): i \geqslant 1\} .
\end{aligned}
$$

By Theorem 53, $\mathcal{P} a l\left(W^{(3)}\right)=\mathcal{C P} a l\left(\bigcup_{i=1}^{4} \mathcal{P}_{i}^{(3)}\right)$.

Example 55. Consider the word $W^{(4)}$. Then by Definition 43 , the sets $\mathcal{P}_{1}^{(4)}, \mathcal{P}_{2}^{(4)}, \mathcal{P}_{3}^{(4)}, \mathcal{P}_{4}^{(4)}$ are as follows:

$$
\mathcal{P}_{1}^{(4)}=\{4 i, 4 i \oplus(010), 4 i \oplus(0102010): i \geqslant 0\},
$$


$\mathcal{P}_{2}^{(4)}=\{4 i \oplus(10201), 4 i \oplus(1020103010201), 4 i \oplus(201030102): i \geqslant 0\}$,

$\mathcal{P}_{3}^{(4)}=\{4 i \oplus(010), 4 i \oplus(01010), 4 i \oplus(0102010), 4 i \oplus(01020102010): i \geqslant 1\}$,

$\mathcal{P}_{4}^{(4)}=\{4 i \oplus(010201030102010), 4 i \oplus(102010301020103010201), 4 i \oplus(00): i \geqslant 1\}$.

By Theorem 53, $\mathcal{P} a l\left(W^{(4)}\right)=\mathcal{C} \mathcal{P} a l\left(\bigcup_{i=1}^{4} \mathcal{P}_{i}^{(4)}\right)$

Example 56. Consider the word $W^{(5)}$. Then by Definition 43 , the sets $\mathcal{P}_{1}^{(5)}, \mathcal{P}_{2}^{(5)}, \mathcal{P}_{3}^{(5)}, \mathcal{P}_{4}^{(5)}$ are as follows:

$$
\begin{aligned}
\mathcal{P}_{1}^{(5)}= & \{5 i, 5 i \oplus(010), 5 i \oplus(0102010), 5 i \oplus(010201030102010): i \geqslant 0\}, \\
\mathcal{P}_{2}^{(5)}=\{ & \{5 i \oplus(10201), 5 i \oplus(1020103010201), 5 i \oplus(10201030102010401020103010201), \\
& 5 i \oplus(201030102), 5 i \oplus(2010301020104010201030102), 5 i \oplus(30102010401020103): i \geqslant 0\}, \\
\mathcal{P}_{3}^{(5)}=\{5 i \oplus(010), 5 i \oplus(01010), 5 i \oplus(0102010), 5 i \oplus(01020102010), 5 i \oplus(010201030102010), & 5 i \oplus(01020103010201030102010): i \geqslant 1\}, \\
\mathcal{P}_{4}^{(5)}= & \{5 i \oplus(00), 5 i \oplus(102010301020104010201030102010401020103010201), \\
& 5 i \oplus(0102010301020104010201030102010): i \geqslant 1\} .
\end{aligned}
$$

By Theorem 53, $\mathcal{P} a l\left(W^{(5)}\right)=\mathcal{C} \mathcal{P} a l\left(\bigcup_{i=1}^{4} \mathcal{P}_{i}^{(5)}\right)$.

\subsection{Length of Palindromes in $W^{(k)}$}

In this section, we want to compute all possible values for the lengths of palindromes in $W^{(k)}$.

Definition 57. Let $k>2$, and for $1 \leqslant i \leqslant 4$. Let $\mathcal{P}_{i}^{(k)}$ be the sets given in Definition 43 . For $1 \leqslant i \leqslant 4$, we define $L\left(\mathcal{P}_{i}^{(k)}\right):=\left\{|P|: U P U^{R} \in \mathcal{P}_{i}^{(k)},|P|>1\right.$ and $\left.U \in \mathbb{N}^{*}\right\}$.

Lemma 58. For each integer $k \geqslant 3$

$$
\begin{aligned}
& L\left(\mathcal{P}_{1}^{(k)}\right):=\left\{2 i-1: 1 \leqslant i \leqslant 2^{k-2}\right\} \\
& L\left(\mathcal{P}_{2}^{(k)}\right):=\left\{2 i-1: 2 \leqslant i \leqslant 2^{k-1}-1\right\} \\
& L\left(\mathcal{P}_{3}^{(k)}\right):=\left\{2 i-1: 2 \leqslant i \leqslant 3 \cdot 2^{k-3}\right\} \\
& L\left(\mathcal{P}_{4}^{(k)}\right):=\left\{2,2 i-1: 2 \leqslant i \leqslant 3 \cdot 2^{k-2}-1\right\} .
\end{aligned}
$$

Proof. We just prove $L\left(\mathcal{P}_{1}^{(k)}\right)=\left\{2 i-1: 1 \leqslant i \leqslant 2^{k-2}\right\}$, the proof of the rest parts are similar to this case. By Definition 43 , it is clear that the set $L\left(\mathcal{P}_{1}^{(k)}\right)$ just contains odd integers. Again by Definition 43, it can be seen that if $t \in L\left(\mathcal{P}_{1}^{(k)}\right)$ is an odd number greater than 2 , then $t-2 \in L\left(\mathcal{P}_{1}^{(k)}\right)$. So if $\ell_{1}$ is the maximum integer in $L\left(\mathcal{P}_{1}^{(k)}\right)$, 
then $L\left(\mathcal{P}_{1}^{(k)}\right)=\left\{1,3,5, \cdots, \ell_{1}\right\}$. Therefore, to prove this part it suffices to show that $\ell_{1}=2^{k-1}-1$. By Definition 43 and Corollary 8 , we have

$$
\ell_{1}=\left|W_{k-1}^{(k)}\right|-1=2^{k-1}-1 .
$$

Theorem 59. For every integer $k \geqslant 3$, the palindrome complexity of $W^{(k)}$ is given by

$$
\operatorname{pal}_{W^{(k)}}(n)= \begin{cases}\infty & \text { if } n \in\{2\} \cup\left\{2 i-1: 1 \leqslant i \leqslant 3 \cdot 2^{k-2}-1\right\} \\ 0 & \text { otherwise }\end{cases}
$$

Proof. Let $A=\{2\} \cup\left\{2 i-1: 1 \leqslant i \leqslant 3 \cdot 2^{k-2}-1\right\}$. Using Theorem 53 and Lemma 58, we find that if $n \notin A$, then there is no palindromic factor in $W^{(k)}$ of length $n$, in other words $\operatorname{pal}_{W^{(k)}}(n)=0$. If $n \in A$, then by Theorem 53 and Lemma $58, W^{(k)}$ has at least one palindromic factor of length $n$ or equivalently $\operatorname{pal}_{W^{(k)}}(n) \neq 0$. By Definitions 43 and 57 , it is easy to see that in this case $\operatorname{pal}_{W^{(k)}}(n)=\infty$.

\section{Acknowledgements}

The authors would like to appreciate anonymous referee for his/her valuable comments which helped to improve the manuscript.

\section{References}

[1] Allouche, J.-P., BaAke, M., Cassaigne, J., and Damanik, D. Palindrome complexity. Theoretical Computer Science 292, 1 (2003), 9-31.

[2] Ambrož, P., Masáková, Z., Pelantová, E., and Frougny, C. Palindromic complexity of infinite words associated with simple Parry numbers. In Annales de l'institut Fourier (2006), vol. 56, pp. 2131-2160.

[3] Berstel, J. Fibonacci words: a survey. In The book of L. Springer, 1986, pp. 13-27.

[4] Berstel, J. Sturmian and episturmian words. (A survey of some recent results). In International Conference on Algebraic Informatics (2007), Springer, pp. 23-47.

[5] DE LuCA, A. A combinatorial property of the Fibonacci words. Information Processing Letters 12, 4 (1981), 193-195.

[6] Droubay, X., Justin, J., and Pirillo, G. Episturmian words and some constructions of de Luca and Rauzy. Theoretical Computer Science 255, 1-2 (2001), $539-553$.

[7] Fischler, S. Palindromic prefixes and episturmian words. Journal of Combinatorial Theory, Series A 113, 7 (2006), 1281-1304.

[8] Glen, A., AND Justin, J. Episturmian words: a survey. RAIRO-Theoretical Informatics and Applications 43, 3 (2009), 403-442.

[9] Glen, A., Simpson, J., And Smyth, W. More properties of the Fibonacci word on an infinite alphabet. Theoretical Computer Science 795 (2019), 301-311. 
[10] Justin, J., And Pirillo, G. On a combinatorial property of Sturmian words. Theoretical Computer Science 154, 2 (1996), 387-394.

[11] Justin, J., AND Pirillo, G. Episturmian words and episturmian morphisms. Theoretical Computer Science 276, 1-2 (2002), 281-313.

[12] Justin, J., And Vuillon, L. Return words in Sturmian and episturmian words. RAIRO-Theoretical Informatics and Applications 34, 5 (2000), 343-356.

[13] Morse, M., And Hedlund, G. A. Symbolic dynamics II. Sturmian trajectories. American Journal of Mathematics 62, 1 (1940), 1-42.

[14] Tan, B., And Wen, Z.-Y. Some properties of the Tribonacci sequence. European Journal of Combinatorics 28, 6 (2007), 1703-1719.

[15] Zhang, J., Wen, Z., And Wu, W. Some properties of the Fibonacci sequence on an infinite alphabet. Electronic Journal of Combinatorics, 24(2) (2017), 2-52, \#P2.52. 